\title{
Hepatocyte organoids and cell transplantation: What the future holds
}

\author{
Weng Chuan Peng ${ }^{1}$, Lianne J. Kraaier ${ }^{1}$ and Thomas A. Kluiver ${ }^{1}$
}

\begin{abstract}
Historically, primary hepatocytes have been difficult to expand or maintain in vitro. In this review, we will focus on recent advances in establishing hepatocyte organoids and their potential applications in regenerative medicine. First, we provide a background on the renewal of hepatocytes in the homeostatic as well as the injured liver. Next, we describe strategies for establishing primary hepatocyte organoids derived from either adult or fetal liver based on insights from signaling pathways regulating hepatocyte renewal in vivo. The characteristics of these organoids will be described herein. Notably, hepatocyte organoids can adopt either a proliferative or a metabolic state, depending on the culture conditions. Furthermore, the metabolic gene expression profile can be modulated based on the principles that govern liver zonation. Finally, we discuss the suitability of cell replacement therapy to treat different types of liver diseases and the current state of cell transplantation of in vitro-expanded hepatocytes in mouse models. In addition, we provide insights into how the regenerative microenvironment in the injured host liver may facilitate donor hepatocyte repopulation. In summary, transplantation of in vitro-expanded hepatocytes holds great potential for large-scale clinical application to treat liver diseases.
\end{abstract}

\section{Introduction}

The expansion and maintenance of primary hepatocytes in vitro while retaining their functional characteristics have been long-standing challenges in the field ${ }^{1}$. Historically, hepatocytes have been cultured in monolayers on collagen gel, but cells quickly lose morphology and the expression of mature hepatic metabolic genes such as cytochromes P450 (CYPs) using this method ${ }^{2-4}$. Various approaches have been employed to promote the viability and function of hepatocytes in vitro, including 2D culture overlaid with Matrigel or collagen gel (termed 'sandwich culture' $^{5}$, cocultures with liver nonparenchymal cell types (e.g., Kupffer cells, hepatic stellate cells [HSCs], or liver sinusoidal endothelial cells [LSECs] $)^{6-8}$ and aggregation into three-dimensional (3D) spheroids $^{9-12}$. While these approaches have resulted in marked improvements in

\footnotetext{
Correspondence: Weng Chuan Peng (w.c.peng@prinsesmaximacentrum.nl) ${ }^{1}$ Princess Máxima Center for Pediatric Oncology, Heidelberglaan 25, 3584 CS Utrecht, The Netherlands

These authors contributed equally: Weng Chuan Peng, Lianne J. Kraaier, Thomas A. Kluiver
}

hepatocyte function and viability, the long-term expansion and maintenance of functional primary hepatocytes remains intractable.

Due to the difficulty in culturing primary hepatocytes, alternative cell sources have been explored, such as the directed differentiation of embryonic stem cells (ESCs) or induced pluripotent stem cells (iPSCs) towards hepatocytes $^{13-16}$. In this context, specification towards the hepatocyte lineage was achieved by employing factors to activate (or inhibit) specific signaling pathways at various time points during culture to mimic in vivo developmental stages ${ }^{17}$. This method allows the generation of large numbers of hepatocyte-like cells. However, the resulting cells often lack functional maturity when compared to their in vivo counterparts ${ }^{18}$.

In addition to ESC/iPSC-derived cultures, major advances have been made in establishing organoids from adult/fetal progenitors or mature cell types derived from healthy primary tissues. In 2009, the Clevers group described the long-term culture of 'mini-intestines' established from leucine-rich repeat-containing G-protein 
coupled receptor 5-positive $\left(\mathrm{LGR} 5^{+}\right.$) crypt base columnar cells $(\mathrm{CBCs})^{19}$. Embedded in 3D extracellular matrix, LGR5 ${ }^{+}$CBCs can be coaxed to self-renew and generate various intestinal cell types in the presence of growth factors such as R-spondin (RSPO), epidermal growth factor (EGF) and noggin (NOG) while spontaneously organizing into crypt-villus structures. Organoid technology has since enabled the in vitro self-renewal of various epithelial cell types, which can be indefinitely expanded while spontaneously self-organizing into 3D structures that resemble their native counterparts ${ }^{16}$. Huch and colleagues described the culture of liver organoids derived from intrahepatic cholangiocytes, i.e., the epithelial cells that form the bile ducts ${ }^{20,21}$. Since then, other groups have shown that organoids can be derived from common bile ducts and gall bladder ${ }^{22-24}$. However, the $3 \mathrm{D}$ culture of primary hepatocytes, which are the principal epithelial cell type that performs most liver metabolic functions, remains elusive. Importantly, the ability to expand primary hepatocytes in vitro is a crucial step towards enabling cell transplantation to treat liver diseases. In a recent breakthrough, the Nusse and Clevers groups described the long-term organoid culture of murine and human primary hepatocytes ${ }^{25,26}$. In this review, we will focus on strategies to derive organoids from primary hepatocytes, drawing insights from signals regulating liver homeostasis and regeneration, on the characteristics of these organoids from the perspective of liver physiology, and finally on the application of hepatocyte organoids for cell transplantation.

\section{Liver homeostasis and repair: insights into culturing hepatocytes in vitro}

The liver is essential for processes such as metabolism, drug detoxification and plasma protein production. The two major parenchymal cell types in the liver are hepatocytes and biliary epithelial cells (BECs; cholangiocytes). Hepatocytes perform most of the liver functions, while bile ducts formed by cholangiocytes transport bile acid produced by hepatocytes to the gallbladder. For an overview of liver architecture, see Fig. 1. The liver is a largely quiescent organ, and hepatocytes are long-lived with an estimated lifespan of $200-400$ days in rodents ${ }^{27-29}$. In addition to diploid hepatocytes, the liver also contains binucleated and polypoid hepatocytes, which appear during postnatal development, increase with aging and are associated with reduced proliferative capacity ${ }^{30}$. Due to its low physiological turnover, the liver does not utilize a dedicated stem cell compartment, akin to the intestinal crypt, to produce new hepatocytes. Rather, new hepatocytes are generated by the existing pool of mature hepatocytes $^{31}$. However, it remains unclear whether all hepatocytes are capable of self-renewal or if specific subsets of hepatocytes are endowed with unique capabilities to divide. Key questions remain: what are the niche signals regulating hepatocyte homeostatic turnover, and what are the defining characteristics of these hepatocytes?

Recent studies employing lineage tracing in mice offer new perspectives on the turnover of hepatocytes in the resting liver. Wang et al. ${ }^{32}$ observed that hepatocytes adjacent to the central vein $(\mathrm{CV})$, under the influence of WNT signals (WNT9B and RSPO3) secreted by the endothelial cells of the $\mathrm{CV}$, preferentially self-renewed and replaced other hepatocytes in the lobule ${ }^{32-34}$. Hepatocytes around the CV expressed WNT target gene axis inhibition protein 2 (Axin2) and progenitor marker T-box transcription factor $3(T b x 3)$, which are normally expressed during fetal liver development ${ }^{32}$. These pericentral hepatocytes are predominantly diploid but can give rise to daughter cells that become polyploid. Over the course of one year of tracing, $20-30 \%$ of the lobule is replaced by daughter cells produced by pericentral hepatocytes. While WNT signaling is predominantly restricted to pericentral hepatocytes in the homeostatic liver, following carbon tetrachloride $\left(\mathrm{CCl}_{4}\right)$-induced liver injury of the pericentral zone, pathway activation is also observed in midlobular hepatocytes surrounding the injury $\operatorname{area}^{35}$. It is perhaps unsurprising that the WNT pathway is implicated in hepatocyte proliferation, given its role in liver development and regeneration ${ }^{34,36,37}$, childhood liver tumors ${ }^{38,39}$ ( $\sim 80 \%$ of hepatoblastoma tumors have activating mutations in CTNNB1, encoding $\beta$-catenin), and in promoting the stem-cell activity of various tissues ${ }^{40,41}$.

In another study, Lin et al. ${ }^{42}$ identified a subset of hepatocytes that expressed high levels of telomerase and were broadly distributed throughout the lobule. In this study, telomerase reverse transcriptase (TERT)-high cells gave rise to small clones (up to eight cells) after 6 months of lineage tracing in the healthy mouse liver ${ }^{42}$. An interesting aspect of TERT-high cells is that they have reduced metabolic activity, suggesting that they may be somewhat adapted for proliferation rather than for regular hepatocyte function. Interestingly, TERT promoter mutations constitute the most frequent genetic alterations in hepatocellular carcinoma, with an overall frequency of $60 \%{ }^{43}$. Of note, several recent studies ${ }^{4-47}$ using various lineage tracing models have argued against the notion that hepatocyte renewal is preferentially localized to either the central or portal zone $e^{32,48}$ but rather is distributed broadly across the lobule. It is also conceivable that multiple and possibly redundant populations of hepatocytes are capable of self-renewal, since the liver is susceptible to various types of damage differently affecting cells in different parts of the lobule ${ }^{49}$.

A recent study of human tissues using retrospective ${ }^{14} \mathrm{C}$ birth dating of cells found that human hepatocytes 
a

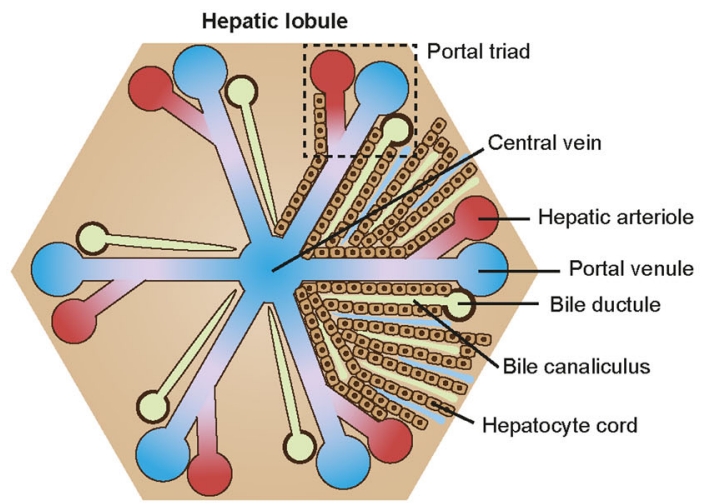

Box 1. Spatial organization of the hepatic lobule

The liver is composed of many repeating hexagonal hepatic lobules, with central veins located at the center of the lobules. At the lobule periphery, multiple portal triads are located where branches of the hepatic artery, portal vein and bile duct are located together. From the central vein, radiating cords of hepatocytes extend outwards towards the periphery of the lobule and the portal triads. Hepatocyte cords form the lining of bile canaliculi, through which bile produced by hepatocytes is transferred to the bile ductules, and of liver sinusoids, fenestrated capillaries where the blood from the hepatic arterioles and portal venules is mixed and flows towards the central vein ${ }^{149}$.

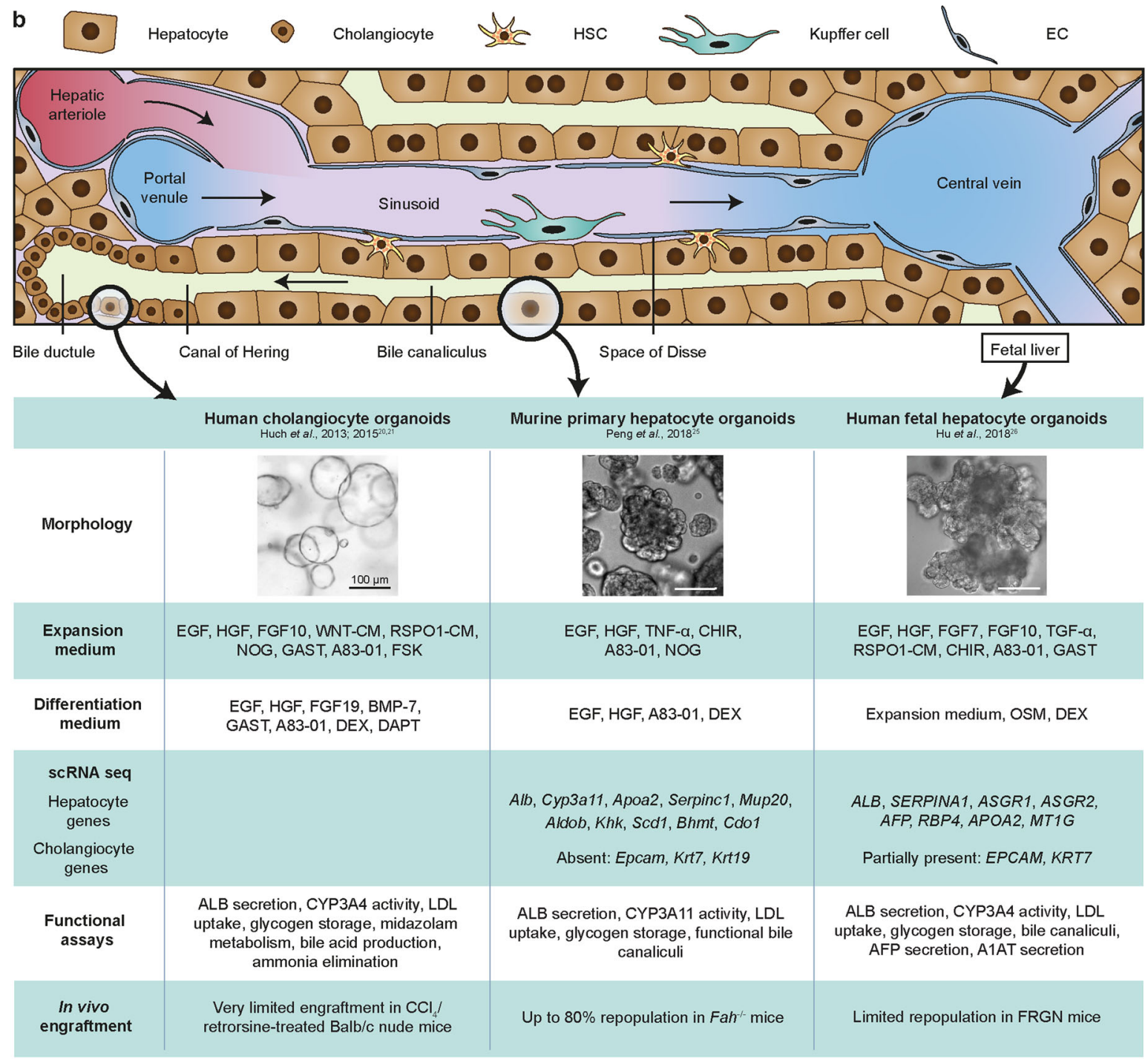

Fig. 1 An overview of the liver lobule and characteristics of organoids derived from either hepatocytes or cholangiocytes. a Schematic diagram of the hepatic lobule showing the portal triad, central vein, and hepatic cords (see also Box $\mathbf{1}^{149}$ ). $\mathbf{b}$ A detailed view of the hepatic lobule showing various cell types in the liver (upper panel) and characteristics of cholangiocyte and hepatocyte organoids derived from either adult or fetal liver $^{20,21,25,26}$ (lower panel). Scale bars: 100 mm; CM: conditioned medium; HSC: hepatic stellate cell; EC: endothelial cell. 
continuously self-renew during the lifetime of an adult to maintain a relatively young liver ${ }^{50}$. Both diploid and polyploid hepatocytes are capable of self-renewal; however, diploid cells have major advantages over polyploid cells in long-term proliferation ${ }^{50}$. This study on human liver tissues is likely more reflective of physiological settings (e.g., regular exposure to chemicals, pathogens, and drug-induced injury) compared to standard laboratory conditions under which mice are maintained. Nonetheless, the above studies in mice and human tissues provide emerging evidence that hepatocytes undergo homeostatic renewal at a more significant rate than previously thought, that hepatocyte proliferation is associated with low ploidy and low metabolic state and that WNT signaling is a likely pathway mediating homeostatic renewal.

\section{Liver regeneration}

In contrast to the low turnover in the resting state, the liver has a unique regenerative capacity, which is crucial for maintaining liver mass following physical or chemical damage $^{31}$. The events that occur after partial hepatectomy $(\mathrm{PHx})$ in rodents have been well described and serve as a basis for understanding the general principles of liver regeneration $^{51,52}$. Notably, regeneration following PHx is not dependent on a small subset of stem cells. Instead, the majority of hepatocytes are able to proliferate ${ }^{53}$.

\section{Regenerative signals}

Following PHx, the plasma levels of EGF, insulin and glucagon (secreted by the intestine, spleen, and pancreas) rise immediately ${ }^{54,55}$. Hepatocyte growth factor (HGF) pro-peptides are released from the extracellular matrix and cleaved by urokinase-type plasminogen activator $(\mathrm{uPA})^{56}$. Together, these molecules act as potent mitogens to promote DNA synthesis. $\mathrm{WNT} / \beta$-catenin signaling is another crucial regulator of liver regeneration, which regulates cell proliferation by promoting G1 phase through cyclin D1 and mitosis ${ }^{57}$. Based on our current understanding of WNT pathway regulation, the LGR5/ $\mathrm{RSPO} /$ zinc and ring finger 3 (ZNRF3) module is likely required for amplifying WNT signals ${ }^{58,59}$. Indeed, this was confirmed by a recent study showing impaired regeneration of the liver in LGR4/5-deficient mice after $\mathrm{PHx}^{34}$. LSECs are the likely source of WNT and RSPO ${ }^{34,60}$. Separately, inflammatory cytokines constitute important early signals that initiate liver regeneration ${ }^{55}$. Two major cytokines that are commonly involved in tissue repair processes across many tissue types are tumor necrosis factor alpha (TNF- $\alpha$ ) and interleukin 6 (IL-6) ${ }^{61}$. TNF- $\alpha$ is produced by liver and spleen macrophages in response to lipopolysaccharide ${ }^{62}$, while IL-6 is secreted by liver macrophages and hepatocytes as a result of TNF- $\alpha$ activation $^{63}$. Other signals that have been implicated in liver regeneration include fibroblast growth factors 7 (FGF-7 $)^{64}$ and 15 (FGF-15; FGF-19 in humans) ${ }^{65}$ and bone morphogenetic protein 7 (BMP-7) ${ }^{66}$. There is likely a redundancy between different signaling pathways, and not all components have been identified ${ }^{67}$.

\section{Intracellular events}

In response to regenerative stimuli, various signaling pathways are activated in hepatocytes that lead to cell cycle progression, DNA synthesis and eventually cell division. Intracellularly, the $\beta$-catenin and $\mathrm{NOTCH}$ intracellular domains (NICD) translocate to hepatocyte nuclei within minutes after PHx. Cytokine-induced transcription factors, such as signal transducer and activator of transcription 3 (STAT-3), nuclear factor kappa-light-chain-enhancer of activated B cells (NF-kB) and activator protein 1 (AP-1), are activated shortly after $^{53}$. Yes-associated protein 1 (YAP1), a critical organ size regulator, is also nuclearly localized $^{68}$. Although no neighboring cells are lost in PHx, the change in shear stress due to increased blood flow activates YAP1 through the Hippo pathway ${ }^{69}$. Hepatocytes secrete various autocrine and paracrine factors, e.g., vascular endothelial growth factor (VEGF), angiopoietin 1 and 2, platelet-derived growth factor (PDGF), FGF-1 and -2 , transforming growth factor alpha (TGF- $\alpha$ ) and granulocyte-macrophage colony-stimulating factor (GM$\mathrm{CSF}$ ), to promote angiogenesis and stimulate the growth of other liver cell types (HSCs, Kupffer cells and biliary cells) ${ }^{63}$. A change in metabolic profile is also observed as a consequence of regeneration. The expression of genes such as alpha-fetoprotein (AFP), hexokinase and fetal isoforms of aldolase and pyruvate kinase, typically absent in the healthy liver, leads to the acquisition of a fetal-like phenotype in the regenerating liver ${ }^{70,71}$.

\section{Termination signals}

When the normal liver mass has been restored, regeneration is gradually terminated. Transforming growth factor-beta (TGF- $\beta$ ) family members, secreted by Kupffer cells and HSCs, act as a brake for hepatocyte proliferation ${ }^{67}$. Another crucial dampening signal is the suppressor of cytokine signaling (SOCS), a negative regulator of various cytokines. In particular, SOCS-1 and SOCS-3, feedback inhibitors of the interleukin (IL)/Janus kinase (JAK)/STAT-3 pathway, inactivate STAT-3 to terminate hepatocyte proliferation ${ }^{72,73}$. Other cytokines, such as interferon-gamma (IFN- $\gamma$ ), have also been shown to downregulate hepatocyte proliferation ${ }^{74}$. Overall, the study of liver regeneration over the years has provided important insights into signals that both activate and terminate hepatocyte proliferation. How hepatocytes reestablish the metabolic gene expression profile of the homeostatic liver upon termination of regeneration remains unclear. 


\section{Regenerative signals for expanding hepatocytes in vitro}

Cholangiocyte organoids have been successfully derived from intra- and extrahepatic cholangiocytes as well as the gallbladder by using combinations of EGF, FGF10, HGF, WNT agonist (WNT3A or CHIR99021), RSPO1, gastrin (GAST), NOG, prostaglandin E2 (PGE2), dexamethasone (DEX), dickkopf-related protein 1 (DKK-1), forskolin (FSK), TGF- $\beta$ inhibitor (A83-01) and rho-associated protein kinase (ROCK) inhibitor $(\mathrm{Y} 27632)^{20-24}$. A related cocktail employing EGF, CHIR99021, A83-01, and Y27632 was able to expand murine primary hepatocytes in a $2 \mathrm{D}$ monolayer $^{75}$. Hepatocellular carcinoma tumor organoid cultures have also been established utilizing the cholangiocyte organoid medium defined by Huch et al. ${ }^{21,76}$. However, such culture media have thus far failed to establish primary hepatocyte organoids, prompting us to hypothesize that the media may lack crucial signals for this purpose.

Drawing insights from liver regeneration in vivo, we speculated that inflammatory signals may be a crucial factor for initiating hepatocyte proliferation in vitro. Indeed, culture medium containing TNF- $\alpha$, along with EGF, HGF, CHIR99021, A83-01, and Y27632, enabled robust proliferation of murine primary hepatocytes ${ }^{25}$. Using this protocol, approximately $15 \%$ of plated hepatocytes were able to form organoids. In the first week of culture, expanded hepatocytes formed small spheroids, which gradually expanded up to several hundred micrometers in the first 3 weeks to form rosette-like structures (Fig. 1b). Notably, they are morphologically distinct from the 'cystic' structures typically observed in cholangiocyte organoids described by Huch et al. and others (Fig. 1b) ${ }^{20,21,23,24}$. While mature hepatocytes are predominantly binucleated or polyploid, we noticed that in vitro-expanded hepatocytes are mostly mononucleated and have a high nucleus-to-cytoplasm ratio, reminiscent of regenerating hepatocytes in the liver following injury ${ }^{25,77}$. The hepatocyte-specific marker hepatocyte nuclear factor 4 alpha (HNF-4 $\alpha$ ) was broadly expressed, and bile canaliculi structures could be detected, reminiscent of the structural polarity typically observed in the liver lobule. Functionally, hepatocyte organoids retain key hepatic functions, such as albumin (ALB) secretion, low-density lipoprotein (LDL) uptake, glycogen storage, and CYP3A11 enzymatic activity, and contain functional bile canaliculi. Furthermore, hepatocyte organoids could upregulate various metabolic genes under appropriate culture conditions. Remarkably, single-cell RNA sequencing (scRNAseq) showed that $A l b$ and Cyp3a11 could be induced to levels comparable to murine primary hepatocytes, indicating that the differentiation capacity is retained even after long-term culture. In vivo, hepatocyte organoids could engraft the injured liver of mice with high efficiency and reestablish the expression profile of zonated markers. Finally, hepatocyte organoids can be serially passaged and expanded for at least 8 months (the longest culture was cryopreserved after approximately 1 year in culture, unpublished data). To our knowledge, this is the first study demonstrating long-term 3D culture of hepatocyte organoids that retain key functional characteristics and in vivo regenerative capacity.

\section{Cytokine dependency and mode of action}

Intriguingly, we found that TNF- $\alpha$ is a crucial factor for the establishment of hepatocyte organoid culture ${ }^{25}$. Substituting TNF- $\alpha$ with IL-6, another inflammatory cytokine, did not yield any organoids. Furthermore, the long-term expansion of hepatocytes is continuously dependent on TNF- $\alpha$. At any point in culture, removal of TNF- $\alpha$ resulted in limited growth of hepatocytes and eventually led to culture deterioration. In vivo, TNF- $\alpha$ is generally considered a crucial signal in liver regeneration. For instance, antibody blockade of TNF- $\alpha$ impaired regeneration ${ }^{78}$, while TNF receptor 1 knockout mice have delayed regeneration ${ }^{79,80}$. The critical role of TNF- $\alpha$ in maintaining in vitro culture is therefore not unexpected.

Earlier hepatocyte culture studies offer some insights into the role of TNF- $\alpha$ in hepatocyte proliferation. A study found that while EGF alone did not promote hepatocyte proliferation in culture, the combination of EGF and TNF- $\alpha$ stimulates proliferation in $\sim 35 \%$ of cultured hepatocytes ${ }^{81}$ by mediating cell entry into $S$ phase through cyclin D1 and cyclin-dependent kinase (CDK) 1 and 2 . In addition, TNF- $\alpha$ suppressed fetal hepatocyte maturation induced by oncostatin $\mathrm{M}$ (OSM) to promote cell cycle progression in culture ${ }^{82}$. TNF- $\alpha$ is also known to prevent apoptosis and promote cell survival and proliferation through NF- $\mathrm{KB}$ signaling ${ }^{83,84}$. Indeed, inhibition of NF- $\mathrm{KB}$ resulted in culture deterioration ${ }^{25}$, indicating that TNF- $\alpha$ signaling is likely mediated by the NF- $k B$ pathway. Importantly, significant crosstalk exists between the NF- $\mathrm{kB}$ and WNT signaling pathways ${ }^{85}$.

A recent study from the Diehl laboratory showed that inflammatory cytokines such as TNF- $\alpha$ can reprogram mature hepatocytes into proliferative hepatocytes with a fetal-like metabolic profile in patients with severe alcoholic hepatitis and in mouse models with liver injury ${ }^{86}$. The acquisition of a fetal profile is mediated by the suppression of the epithelial splicing regulatory protein 2 (ESRP2)-mediated adult splicing program, resulting in the accumulation of fetal splicing variants of genes such as NF2 and CSNK1D, which have reduced kinase activity for YAP1 degradation. Consequently, YAP1 target genes such as AREG, CTGF, and PTGS2 were upregulated. Consistent with in vivo observations, qRT-PCR and splice isoform analysis of hepatocyte organoids cultured in TNF- $\alpha$ medium $^{25}$ revealed significant downregulation of 
Esrp2, accumulation of fetal RNA-splicing variants ( $N f 2$, Slk, Fln, and Kras) and upregulation of the aforementioned YAP1 target genes ${ }^{25}$. Importantly, upon TNF- $\alpha$ withdrawal, hepatocyte organoids reestablish Esrp2 expression and upregulate adult liver-specific markers such as ammonia detoxification pathways and blood coagulation factors. In sum, this study provides a potential mechanism through which TNF- $\alpha$ reprograms adult hepatocytes into fetal-like hepatocytes ${ }^{86}$.

\section{Cytokine-induced in vitro regeneration beyond the liver}

The liver is not the only organ that is dependent on inflammatory signals for regeneration after injury. Indeed, across multiple tissues, for example, skeletal muscle, intestine, colon, hair follicles, skin, and the central nervous system, inflammation and regeneration are tightly coupled ${ }^{87,88}$. The inflammatory cytokines TNF- $\alpha$ and interleukins trigger regenerative responses in adult progenitors or mature cells by activating a multitude of transcription factors (e.g., NF-kB, JAK/STAT, AP-1, YAP1, and $\mathrm{NOTCH}$ ), thereby activating a transcriptional program that promotes cell survival, proliferation, dedifferentation and acquisition of a fetal-like phenotype $^{88}$. Such observations led us to postulate that regenerative cytokines may have a more general role in cell cycle regulation and could potentially be harnessed beyond the context of injury to expand otherwise quiescent/slow-cycling primary cell types in vitro. Indeed, the regenerative effect of cytokines in vitro has also been employed to expand other tissue types, i.e., muscle and lung, both of which are known to undergo cytokinedriven regeneration following injury ${ }^{89-91}$. Indeed, muscle stem cells (satellite cells) can be expanded long-term with a combination of cytokines (TNF- $\alpha$, IL-1 $\alpha$, IL-13, and IFN- $\gamma$ ), while lung alveolar type 2 epithelial cells robustly proliferate in the presence of IL-1 and TNF- $\alpha$. Collectively, these studies provide emerging evidence supporting the role of cytokines in the in vitro culture of otherwise 'hard-to-culture' cell types.

\section{An alternative strategy: fetal-derived human hepatocytes}

In a parallel study by the Clevers group, $\mathrm{Hu}$ et al. ${ }^{26}$ expanded murine hepatocytes using a cocktail that included EGF, HGF, FGF7 and 10, WNT agonists (CHIR99021 and RSPO1), A83-01 and Y27632 (Fig. 1b). FGF7 is a niche factor previously found to be important for liver regeneration ${ }^{92}$. Interestingly, $\mathrm{Hu}$ et al. ${ }^{26}$ observed that cells around the CV have a slightly higher organoidforming efficiency than the rest of the hepatocytes in the lobule. Diploid and tetraploid hepatocytes, but not octaploid cells, can form organoids. Morphologically, hepatocyte organoids resemble 'grape-like' clusters of cells, as opposed to the more densely packed structures observed in hepatocyte organoids established by Peng et $\mathrm{al}^{25}$. Importantly, hepatocyte organoids were able to perform major functions, such as ALB secretion, CYP1A2 activity, glycogen storage and LDL uptake, and displayed extensive bile canaliculi networks. While the cocktail media was able to support organoid establishment, only a small fraction of plated cells formed hepatocyte organoids $(0.5-1 \%)$. The rate of organoid formation in this medium is considerably lower than that in media with TNF- $\alpha$. Notably, organoids could be maintained for up to 3 months and showed limited growth afterwards ${ }^{26}$.

To culture human hepatocytes, $\mathrm{Hu}$ et al. ${ }^{26}$ adopted a different strategy by using fetal-derived liver cells, which are expected to have higher proliferative capacities than mature hepatocytes and hence are more suitable for longterm propagation. Previous studies indicated that fetal hepatocytes could be maintained in culture for several months while acquiring mature hepatic function ${ }^{93,94}$. In the current study, fetal liver cells were obtained from donor fetuses at 11-20 weeks of gestation using a two-step perfusion method that typically enriches hepatocytes in the adult liver. Approximately $1 \%$ of plated cells were able to form organoids ${ }^{26}$. The identity of the cells (or their corresponding developmental stages) that gave rise to organoids was not determined in this study. Using a medium similar to that of murine organoid culture, human fetal liver-derived hepatocyte organoids could be expanded for close to a year. Transmission microscopic imaging showed the accumulation of glycogen particles and revealed cellular features (large nuclei with prominent nucleoli, large numbers of mitochondria with few cisternae, bile canaliculi, tight junctions, etc.) that closely resemble hepatocytes, indicative of maturation in vitro ${ }^{26}$.

\section{Adult human hepatocyte culture remains challenging}

In the same study, $\mathrm{Hu}$ et $\mathrm{al}^{26}$ established hepatocyte organoids using human hepatocytes from adult and pediatric sources. However, when compared to fetalderived hepatocytes or adult mouse primary hepatocytes, the proliferative capacity of mature hepatocytes appeared limited (2-2.5 months). Since the publication of our work, several groups have reported monolayer cultures of human hepatocytes ${ }^{95-100}$. Zhang et al. ${ }^{96}$ found that the expression levels of senescence and cell cycle arrest genes increased markedly during culture, and the growth of adult hepatocytes was arrested after 4 passages (which corresponds to $\sim 300$-fold expansion). To counter this cellular senescence effect, cells were cultured under hypoxic conditions and consequently showed a 10,000-fold expansion (up to 8 passages). Separately, Katsuda et al. ${ }^{100}$ attempted to reprogram adult human hepatocytes based on their previously described small-molecule-mediated culture 
method. They found that adult human hepatocytes showed very limited expansion. Next, Katsuda et al. focused on infant hepatocytes, which have been shown to be easier to expand in culture ${ }^{101}$. Upon treatment with the maturationinducing factors OSM and DEX, these cells could be differentiated into cells expressing several functional CYP enzymes (e.g., CYP1A2, CYP2B6, and CYP3A4) at levels comparable to mature hepatocytes. Furthermore, they could be passaged for over 10 passages, although the expression of hepatic markers and functional activities (such as CYP enzymatic activities) gradually declined during culture, as noted by the authors. These studies, as well as other recent studies, highlighted the challenges in expanding adult human hepatocytes long-term ${ }^{95-100}$.

\section{Cell identity and state: bipotent or not?}

The identity of cultured hepatocytes in vitro has always been an intriguing question, since earlier studies found that hepatocytes can adopt a biliary fate following injury and in vitro culture ${ }^{67,102}$. The first evidence for hepatocyte to cholangiocyte transition in vivo was shown by Michalopoulos et al. ${ }^{103}$, who demonstrated that dipeptidyl peptidase IV (DPPIV) ${ }^{+}$-transplanted hepatocytes acquired a ductular morphology upon induction of bile duct injury in the DPPIV ${ }^{-}$host rat model. In a later study, Yanger et al. ${ }^{104}$ used lineage tracing in mouse cholestatic liver injury models to demonstrate that hepatocytes can undergo transition into ductal-like cells upon cholangiocyte damage. Moreover, Schaub et al. ${ }^{105}$ demonstrated that in a mouse model of human Alagille syndrome, a disease characterized by impaired formation of peripheral bile ducts, a partially functional biliary tree is still formed. The researchers used lineage tracing to show that these ducts were of hepatocyte origin and that hepatocytes were capable of de novo bile duct formation in this disease model. Together, these studies demonstrate the capability of hepatocytes to transition to a ductal state when cholangiocyte proliferation is suppressed.

Single-cell RNA-seq allows for the evaluation of the heterogeneity among cultured hepatocytes. Based on scRNA-seq, Hu et al. ${ }^{26}$ identified five distinct clusters of cells in their murine hepatocyte cultures, which they defined as noncycling mature hepatocytes $\left(A l b^{\text {high }}\right)$, cycling progenitor cells with high levels of progenitor markers (secreted phosphoprotein $1[S p p 1]^{\text {high }}, A l b^{\text {low }}$ ), more primitive progenitor cells with high levels of cycling genes and lacking mature markers (two clusters), and some cells with biliary markers (keratin $7[\mathrm{Krt} 7]^{+}$). Consistent with the transcriptomic data, cystic organoids that resemble biliary organoids formed spontaneously in culture from $\mathrm{ALB}^{+}$-labeled cells. Furthermore, hepatocytes cultured in biliary media strongly upregulate archetypal biliary markers (KRT7 and KRT19), while hepatocyte markers (HNF-4 $\alpha$ and ALB) are repressed.
The hepatocyte to biliary conversion is consistent with previous studies in monolayer culture ${ }^{75,102}$. Using small molecules (CHIR99021, Y27632 and A83-01), Katsuda et al. ${ }^{75}$ demonstrated that mature hepatocytes can be reprogrammed into bipotent progenitors and can be induced to upregulate either hepatocyte or biliary markers. Under culture conditions promoting biliary fate, biliary markers such as KRT19, aquaporins (AQPs) 1 and 9, and ion channel proteins cystic fibrosis transmembrane conductance regulator (CFTR) and anion exchange protein 2 (AE2) were strongly induced. The resulting ductal-like structures secrete fluorescein dye into the luminal space and can respond to the hormone secretin by enlarging their luminal space, indicative of functional BECs.

Separately, scRNA-seq by Peng et al. ${ }^{25}$ revealed broad expression of hepatocyte-specific markers (e.g., $H n f-4 \alpha$, Alb, apolipoprotein A1 (Apoa1), transthyretin (Ttr), and Serpina1c) across the entire population of cultured hepatocytes. Cultured cells were enriched in genes that are related to cytolysis, lipoprotein particle remodeling, blood coagulation, retinol metabolic process, regulation of G1/S phase transition, and response to tumor necrosis factor. In addition, a subset of cells highly expressed genes that are related to mitosis, cell cycle regulation, chromosome segregation, remodeling and DNA replication, indicative of cycling hepatocytes. One major difference from other in vitro cultures is the absence of canonical biliary markers (such as epithelial cell adhesion molecule [Epcam], Krt7, Krt19, Aqp1, Aqp4 and claudin 7 [Cldn7]), indicating that the vast majority of cells cultured with TNF- $\alpha$ are hepatocytes, without evidence of transdifferentiation. Taken together, the above studies demonstrated that hepatocytes in culture display cellular plasticity, and the biliary fate can be suppressed by adding appropriate signals, yielding solely hepatocytes.

\section{'Proliferative' vs 'metabolic' states}

An interesting aspect of hepatocyte organoids is that in vitro culture recapitulates some aspects of liver regeneration ${ }^{25,26}$. This is supported by RNA-seq data comparing hepatocyte organoids with hepatocytes isolated from livers subjected to $\mathrm{PHx}^{26}$. We noted that transcription factors typically associated with tissue regeneration and previously described to be critical for liver regeneration after $\mathrm{PHx}$ were upregulated ex vivo ${ }^{88,106,107}$. For instance, the transcription factors Rela (NF-кB p65 subunit), Stat3, Yap1, Myc, Jun and Fos (which form the AP-1 complex) were detected in organoids but only at low levels or absent in primary hepatocytes isolated from healthy mouse livers. Acute phase proteins, as well as cytokines and chemokines associated with inflammatory responses, were highly expressed in proliferating hepatocytes in vitro. In addition, hepatocyte 


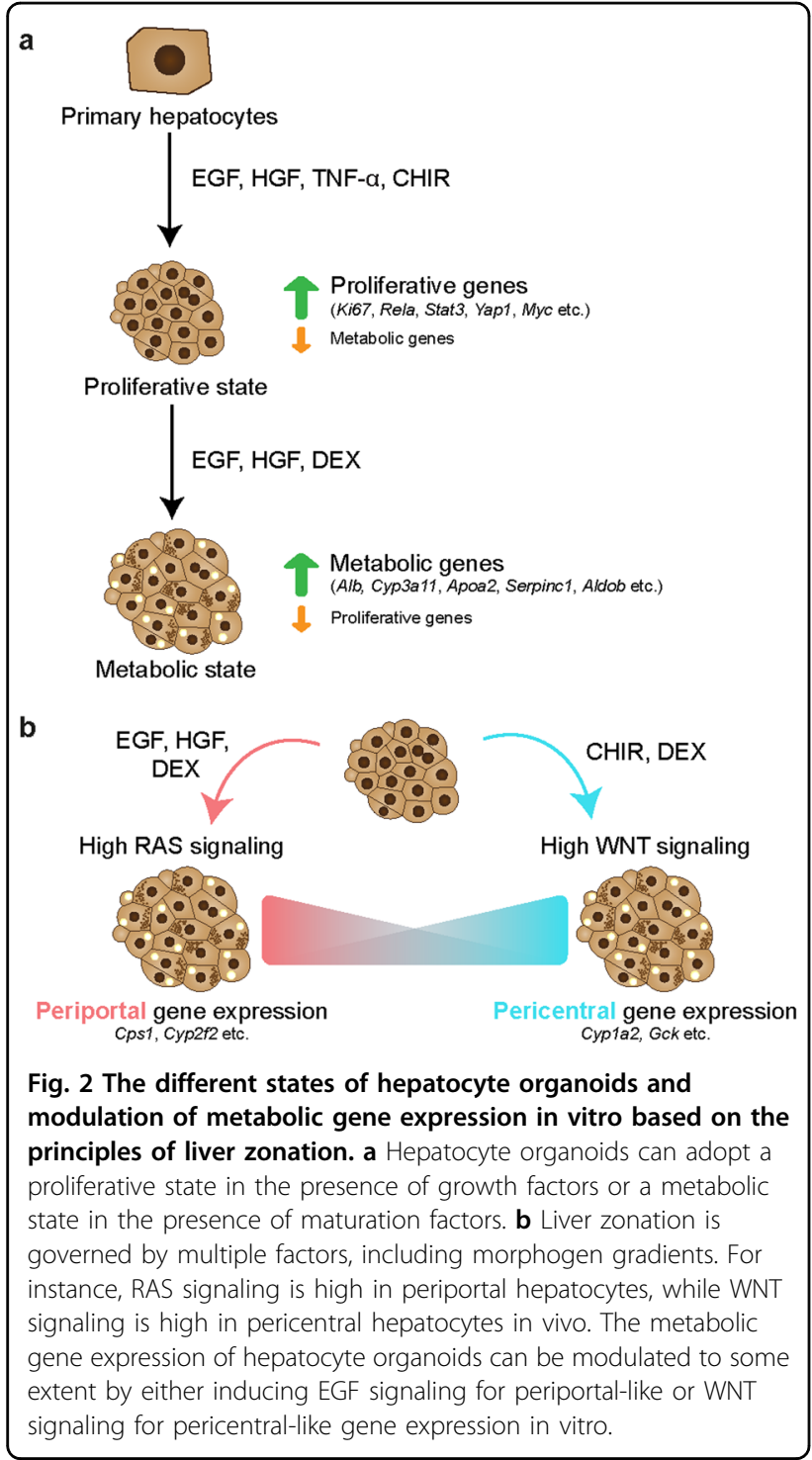

organoids secrete growth factors, including insulin-like growth factor 2 (IGF2), TGF- $\alpha$ and angiogenic factor VEGF-A ${ }^{108-110}$.

A major consequence of 'in vitro regeneration' is the downregulation of metabolic genes that are typically abundantly expressed in the liver, suggesting an adaptation to a 'proliferative' state (Fig. 2a). For instance, CYP enzymes (Cyp3a11 and Cyp2e1), apolipoproteins (Apoa2 and Apoc3), serine protease inhibitors (Serpin1e and Serpin $3 k$ ) and major urinary proteins (Мup3 and Mup20) were expressed at low levels compared to adult murine hepatocytes ${ }^{25}$. To test whether proliferating hepatocytes reexpress functional genes upon withdrawal of expansion and inflammatory factors, WNT and TNF- $\alpha$ were withdrawn from the culture medium, and DEX was added to promote hepatocyte maturation (see also the section below). Indeed, when hepatocytes cultured in expansion medium (for 2-3 months) were switched to differentiation medium for 3-5 days, cultured cells upregulated suites of genes that correspond to mature hepatocyte functions. scRNA-seq showed that hepatocyte markers such as Alb (the most abundant plasma protein), Cyp3a11, Serpina1, Aldob, Khk, Bhmt and Mup20 were induced to levels comparable to those in primary hepatocytes. In addition, functional assays, such as albumin secretion and CYP3A11 enzymatic activity, performed on hepatocyte organoids (at 3-7 months in culture) corroborated the scRNA-seq data. Concomitantly, markers that are associated with regeneration and proliferation were downregulated, correlating with reduced proliferation of organoids in the differentiation medium ${ }^{25}$.

A recent single-cell profiling study surveying the transcriptomic landscape of hepatocytes following $\mathrm{PHx}$ provides evidence that hepatocytes transiently reactivate a postnatal-like transcriptomic program to undergo an intense phase of proliferation, with a distinct subset of cells becoming 'metabolically hyperactive' to compensate for any temporary deficits in liver function ${ }^{111}$. This division of labor ensures that the liver continues to function optimally while undergoing tissue repair. Taken together, these studies show that hepatocytes can adopt either a 'proliferative state' or 'metabolic state', both in vivo and in vitro (Fig. 2a). More importantly, hepatocyte organoid cultures retain the ability to upregulate the expression of various metabolic markers typically seen in mature hepatocytes. However, it should be noted that the metabolic profile of the adult liver is highly zonated and regulated by multiple factors, many of which are not present in the current culture conditions ${ }^{25,26,75}$. In the following section, we discuss how the understanding of pathways regulating liver zonation and niche signals present in the liver microenvironment can be exploited to modulate the hepatocyte expression profile in vitro.

\section{Modulating hepatocyte function in vitro: from the perspective of liver zonation}

Hepatocytes within the liver sinusoid are exposed to varying gradients of morphogens, oxygen, nutrients, hormones and metabolites, resulting in zonated gene expression, whereby cells around the portal vein (PV) express different subsets of genes from cells around the $\mathrm{CV}^{112,113}$. The $\mathrm{WNT} / \beta$-catenin pathway is generally considered one of the major determinants of spatial zonation of gene expression and termed the 'zonation keeper'114. Production of WNT9B and RSPO3, as well as WNT2 expressed in sinusoidal endothelial cells extending from the $\mathrm{CV}$, results in a WNT morphogen gradient in the lobule. By contrast, WNT/ $\beta$-catenin activity is repressed by the negative regulator adenomatous polyposis coli (APC) in the portal region ${ }^{114}$. As Benhamouche et al. ${ }^{114}$ showed, blockade of WNT signaling promotes periportal 
gene expression, and knocking out Apc promotes pericentral gene expression. While WNT ligands are enriched in the pericentral region, RAS signaling has been postulated to be one of the major pathways regulating periportal gene expression (Fig. 2a) ${ }^{113,115}$. In addition to the RAS pathway, glucagon has been shown to regulate liver zonation by opposing the WNT pathway ${ }^{116}$. Likely, many other pathways that may contribute to liver zonation remain to be identified.

In our recent study ${ }^{25}$, we found that the expression levels of zonated genes (e.g., carbamoyl-phosphate synthase $1[C p s 1]$ and Cyp2f2) were low in the expansion media but could be modulated in vitro. To induce periportal genes such as Cps1 and Cyp2f2, a medium containing EGF, HGF and DEX (without WNT and TNF- $\alpha$ ) was devised. DEX, a corticosteroid that exerts antiinflammatory effects on hepatocytes, has been previously shown to suppress hepatocyte proliferation and promote differentiation in culture ${ }^{117}$. Another medium containing the WNT agonist CHIR99021 and DEX (without EGF, HGF and TNF- $\alpha$ ) was employed to promote pericentral genes such as Cyp1a2 and glucokinase $(G c k)^{25}$. Notably, the expression of these aforementioned genes could be massively induced (up to several thousand-fold) (Fig. 2b). In addition to hepatocytes, nonparenchymal cell types in the liver also display a zonated pattern of expression $^{118,119}$. Halpern et al. ${ }^{119}$ showed, by sequencing closely associated pairs of hepatocytes and endothelial cells, that endothelial cells displayed profound spatial heterogeneity, with one-third of the genes being significantly zonated. For instance, Wnt9b and Rspo3 are enriched in the endothelium of the CV, while Bmp2 and Stab1 are repressed. Spatially localized niche signals, either by endothelial cells or other cell types in the liver, likely contribute to the zonated expression pattern of hepatocytes. We anticipate the incorporation of these niche factors in the culture media to improve the expression of zonated genes in hepatocyte organoids.

While it is desirable to recreate liver zonation in vitro to achieve functionally diverse hepatocytes in culture, this has been technically challenging due to the absence of spatially localized niche signals and morphogen gradients typically observed in native tissue. Furthermore, the WNT protein has traditionally been difficult to manipulate due to its insolubility and poor expression yield. Major advances have been made in the field through the engineering of potent soluble WNT agonists ${ }^{120,121}$, which are more amenable to manipulation. WNT agonists are engineered to engage LRP6 and FZD receptors, for instance, by linking a pan-Frizzled antibody to the Cterminal domain of the WNT antagonist DKK1. The use of WNT agonists for culturing various types of epithelial organoids has been established recently ${ }^{121}$. Specifically, we showed that hepatocyte organoids can be robustly expanded using next-generation WNT surrogates, following the protocol described previously ${ }^{25,121}$. Separately, earlier work by Habib et al. ${ }^{122}$ showed that immobilized WNT, i.e., WNT tethered to beads, can act as a stem cell niche to mediate asymmetric division ${ }^{123,124}$. In a recent study, the Lutolf laboratory showed that microstructured biological scaffolds that mimic the crypt-villus structure can be used to maintain the mini-intestine in vitro ${ }^{125}$. Collectively, micropatterned scaffolds, together with coculture with nonparenchymal cell types, tethering of WNT agonists to a 'signaling center' mimicking the CV, and modulation of morphogen flow, may enable hepatocyte culture with spatial organization and gradient gene expression that resembles the liver lobule.

\section{Hepatocyte transplantation to treat liver diseases}

Orthotopic liver transplantation is currently the favored treatment option for end-stage chronic liver diseases. Due to severe donor organ shortages, hepatocyte transplantation has been explored as an alternative strategy. Cryopreserved primary human hepatocytes have been used for transplantation in patients with various types of diseases, such as acute liver failure ${ }^{126}$, factor VII deficiency ${ }^{127}$ and Crigler-Najjar syndrome ${ }^{128-130}$ (reviewed by Barahman et al. $2018^{131}$ ). However, the lack of good-quality donor cells, limited engraftment efficiency, immune rejection of transplanted cells despite the application of immunosuppression, and the loss of clinical benefit over time have precluded large-scale clinical applications of hepatocyte transplantation. Here, we discuss the types of liver diseases that can potentially be treated by cell transplantation, the potential use of in vitro-expanded hepatocytes for cell transplantation, and strategies to improve their long-term engraftment.

\section{Types of liver diseases that can potentially be treated by hepatocyte transplantation}

The efficiency of repopulation by hepatocytes in the host liver and the extent of liver repopulation needed for therapeutic benefit vary between different types of liver diseases, depending on the pathophysiology of the diseased host liver (Fig. 3). For instance, many liver-based disorders are caused by a single gene defect, including Crigler-Najjar syndrome ${ }^{128-130}$ (hyperbilirubinemia), factor VII deficiency ${ }^{127}$ (blood clotting disorder) and phenylketonuria ${ }^{132}$ (phenylalanine accumulation in blood). The absence of functional gene products prevents certain essential hepatic functions from being carried out without damaging the liver itself. In this context, small numbers of engrafted hepatocytes can provide therapeutic benefits and alleviate disease symptoms by the production of the necessary functional proteins. The number of engrafted donor cells needed for clinical benefit for these diseases is unknown, but several patients showed reduced disease 


\begin{tabular}{|c|c|c|}
\hline $\begin{array}{l}\text { Liver-based disorders } \\
\text { without hepatocyte injury }\end{array}$ & $\begin{array}{l}\text { Liver-based disorders } \\
\text { with hepatocyte injury }\end{array}$ & cute liver failure \\
\hline $\begin{array}{l}\text { E.g. Crigler-Najjar syndrome }{ }^{128,129,130} \text {, Factor } \\
\text { VII deficiency }{ }^{129} \text {, phenylketonuria }{ }^{130}\end{array}$ & $\begin{array}{l}\text { E.g. tyrosinemia type } 1^{152}, a 1 \text {-antitrypsin } \\
\text { deficiency }\end{array}$ & $\begin{array}{l}\text { E.g. mushroom poisoning }{ }^{128} \text {, toxicity (alcohol, } \\
\text { drugs), viral hepatitis }{ }^{154}\end{array}$ \\
\hline $\begin{array}{l}\text { In Crigler-Najjar syndrome, severe } \\
\text { unconjugated hyperbilirubinemia results in } \\
\text { increased risk of brain damage, without affecting } \\
\text { hepatic architecture and function }{ }^{128} \text {. }\end{array}$ & $\begin{array}{l}\text { In tyrosinemia type } 1, \text { FAH deficiency results } \\
\text { in accumulation of a toxic metabolite during } \\
\text { tyrosine breakdown causing hepatocyte injury }{ }^{152} \text {. }\end{array}$ & $\begin{array}{l}\text { Generally, acute liver failure is characterized } \\
\text { by rapid loss of liver mass and function and can } \\
\text { lead to multi-organ failure }{ }^{155} \text {. }\end{array}$ \\
\hline $\begin{array}{l}\text { Donor hepatocytes can engraft but do not } \\
\text { extensively repopulate the liver parenchyma. } \\
\text { Donor cells do not have a proliferative advantage } \\
\text { over host hepatocytes }^{150.151} \text {. }\end{array}$ & $\begin{array}{l}\text { Donor hepatocytes can engraft and extensively } \\
\text { repopulate the liver parenchyma. } \\
\text { Donor hepatocytes have a proliferative advantage } \\
\text { over injured host hepatocytes }{ }^{140} \text {. }\end{array}$ & $\begin{array}{l}\text { Host liver regeneration can occur upon } \\
\text { auxiliary orthotopic liver transplantation, } \\
\text { depending on the severity of the injury }{ }^{156} \text {. }\end{array}$ \\
\hline $\begin{array}{l}\text { Engraftment of small numbers of hepatocytes } \\
\text { can provide therapeutic benefit by alleviating } \\
\text { disease symptoms }{ }^{128.129 .130} \text {. }\end{array}$ & $\begin{array}{l}\text { Abundant repopulation by donor hepatocytes } \\
\text { resolves liver injury and restores normal liver } \\
\text { function }^{139.140} \text {. }\end{array}$ & $\begin{array}{l}\text { Engrafted hepatocytes support liver } \\
\text { function before liver transplantation can be } \\
\text { performed }{ }^{157} \text {. }\end{array}$ \\
\hline
\end{tabular}

Fig. 3 An overview of the types of liver diseases that can potentially be treated with hepatocyte transplantation ${ }^{128-130,139,140,150-157}$. The efficiency of engraftment and repopulation of donor hepatocytes is dictated by the host tissue microenvironment. In addition, the number of donor hepatocytes needed to provide therapeutic benefit is dependent on the liver pathophysiology.

symptoms after receiving cell numbers corresponding to $\sim 5-10 \%$ of the liver mass ${ }^{131}$. For instance, transplantation of $\sim 4 \%$ of the total liver mass in a patient with CriglerNajjar syndrome resulted in greatly decreased serum bilirubin levels, which were maintained for a period of six months, after which disease remission occurred, and the patient eventually received a whole liver transplant ${ }^{130}$. The loss of clinical benefit over time, however, suggests the lack of long-term donor cell engraftment in the host liver.

\section{Cell transplantation with in vitro-expanded hepatocytes}

To improve the clinical outcome of hepatocyte transplantation in humans, major challenges need to be addressed, such as the lack of healthy and good-quality primary cells suitable for transplantation, difficulty in preservation, low engraftment and repopulation and lack of long-lasting effects on the restoration of organ functions ${ }^{130,131,133}$. Cryopreserved primary human hepatocytes have thus far been used for clinical applications of hepatocyte transplantation. While cryopreservation facilitates long-term storage of donor material, cryopreserved hepatocytes showed reduced cell viability, cell attachment efficiency and albumin production compared to freshly isolated primary hepatocytes, leading to compromised quality for cell transplantation ${ }^{133}$. Cultured hepatocytes tolerate cryopreservation better than freshly isolated primary hepatocytes. Rodent hepatocytes that were cultured for $24 \mathrm{~h}$ in a monolayer prior to cryopreservation displayed higher viability and plating efficiencies upon thawing than primary hepatocytes that were cryopreserved directly after isolation ${ }^{134}$. Based on our own experiences, hepatocyte organoids can be freeze-thawed repeatedly for in vitro expansion. Cultured hepatocytes ${ }^{25}$ could be shipped overnight on ice in expansion medium supplemented with $2 \%$ fetal bovine serum and retained high viability ( $>90 \%$ viability, our unpublished data) for transplantation in mice.

\section{Engraftment of in vitro-expanded primary hepatocytes in liver injury mouse models Cholangiocyte-derived organoids}

Huch et al. ${ }^{20}$ showed that biliary organoids derived from intrahepatic cholangiocytes could be induced to express hepatocyte markers (e.g., HNF-4 $\alpha$, ALB and multidrug resistance-associated protein 4 (MRP4)) and display some hepatic functions (e.g., LDL uptake and glycogen storage) in vitro. However, these 'transdifferentiated' biliary organoids showed limited repopulation in fumarylacetoacetate hydrolase-deficient $\left(\mathrm{Fah}^{-l-}\right)$ mice $(<1 \%$ of the liver parenchyma $)^{20}$. Similarly, human biliary organoids only engrafted as singlets and doublets into the mouse liver and showed no evidence of repopulation in BALB/c nude mice $^{21}$. Such low repopulation of the liver by biliary organoids is most likely a result of poor transdifferentiation of biliary cells to hepatocytes in vitro prior to transplantation and/or in vivo following transplantation. In line with this, cholangiocyte to hepatocyte transdifferentiation is rarely observed during liver regeneration in vivo ${ }^{135,136}$ but only under extreme circumstances where hepatocyte proliferation is severely impaired in genetically modified mouse models ${ }^{137}$. The lack of engraftment and repopulation using cholangiocyte-derived organoids 
demonstrated the need for using primary hepatocyte organoids as the cell type for transplantation.

\section{Primary hepatocyte organoids}

The in vivo regenerative capacity of in vitro-expanded primary hepatocytes was demonstrated by transplantation of murine hepatocyte organoids into $\mathrm{Fah}^{-1-}$ mice ${ }^{25}$. Engrafted hepatocytes massively repopulated the injured host liver at approximately 100 days posttransplantation, resulting in occupation of up to $80 \%$ of the liver parenchyma by $\mathrm{FAH}^{+}$clones of various sizes. Donor cells expressed the hepatocyte marker HNF-4 $\alpha$ but not the biliary markers SOX9, KRT7 or KRT19. Of note, hepatocyte organoids were cultured in media promoting expansion prior to transplantation. Intriguingly, donor cells underwent maturation in vivo and reestablished expression of zonation markers. For instance, cells adjacent to the CV expressed markers such as glutamine synthetase (GS), glutamate transporter 1 (GLT1) and CYP2E1, while cells outside the CV areas expressed CPS1. Notably, the expression of these markers was not detected in the host tissue $^{25}$. Overall, these observations suggest that the expression profile of donor hepatocytes upon transplantation is dictated by the host liver microenvironment.

\section{Hepatocyte organoids derived from human fetal liver}

$\mathrm{Hu}$ et al. ${ }^{26}$ were able to successfully engraft human fetal liver-derived hepatocyte organoids in $\mathrm{Fah}^{-1-} / \mathrm{Rag}^{-/-} / \mathrm{Il}-$ $2 r \gamma^{-1-}$ (FRG)N mice (i.e., FRG-deficient mice in a nonobese diabetic (NOD) strain), albeit to a limited extent. Nonetheless, engrafted cells expressed functional markers, such as ALB, CYP2E1 and MRP2, but not the fetal marker AFP, indicative of in vivo maturation. The reason for the limited extent of engraftment is unclear and could be attributed to the lack of maturation in vivo. To test this hypothesis, the authors compared the engraftment of fetalderived organoids with primary hepatocytes and organoids obtained from one pediatric source and noted that primary hepatocytes and organoids significantly outperformed fetal-derived organoids in terms of engraftment and proliferation $^{26}$. This result suggests that mature hepatocytes are better suited for cell transplantation.

\section{D culture of primary murine/human hepatocytes}

In addition to in vitro-expanded primary hepatocyte organoids, an earlier study by Katsuda et al. ${ }^{7,100}$ showed that chemically induced liver progenitors (CLiPs) generated from rat hepatocytes retained high in vivo repopulation capacity. Using cDNA-uPA/severe combined immunodeficient (SCID) mice, which express uPA specifically in hepatocytes under the albumin promotor to induce severe liver injury, CLiPs repopulated up to $90 \%$ of the host livers and expressed HNF-4 $\alpha$, multidrug resistance-associated protein 2 (MRP2) and various CYP enzymes, indicative of substantial in vivo differentiation $^{75,100}$. Building upon their previous work ${ }^{75}$, Katsuda et al. ${ }^{100}$ showed that human infant hepatocytes reprogrammed in culture to human CLiPs displayed highly variable repopulation efficiency in TK-NOG and cDNAuPA/SCID mice. In some mice, repopulation of more than $\sim 90 \%$ of the liver parenchyma was achieved, and cells expressed the functional markers MDR1, TTR, GS, CYP1A2, and CYP3A4, indicating maturation in vivo. In another study, Zhang et al. ${ }^{96}$ showed that cryopreserved human hepatocytes that were expanded in vitro could substantially repopulate the livers of FRG mice. Donor cells expressed the hepatocyte markers HNF-4 $\alpha$, ALB, CYP3A4, and GS ${ }^{96}$. Notably, the latter two studies showed that the repopulation capacity of expanded human hepatocytes decreased upon longer in vitro culture, as cells at later passages showed reduced repopulation after transplantation when compared to earlier passages ${ }^{96,100}$. These studies suggest that suboptimal culture conditions may have somewhat compromised the engraftment potential of the cells.

\section{Regenerative host liver microenvironment facilitates donor cell repopulation}

How hepatocytes repopulate the host liver remains an intriguing question. Previous studies by the Grompe laboratory and others demonstrated that transplanted primary hepatocytes could replace up to $90 \%$ of the mouse host liver of Fah-deficient ${ }^{138}$ or FRG-deficient ${ }^{139}$ mice, which are widely used to study liver repopulation (Fig. 4a). Given that $\sim 1$ million cells (or $<2 \%$ of total liver mass) are typically transplanted into mice and only $\sim 10 \%$ of the injected cells are estimated to engraft in the liver ${ }^{139}$, significant expansion in vivo needs to occur to achieve the levels of repopulation observed. In these studies, $\mathrm{Fah}^{-1-}$ mice were continuously administered the drug 2-(2-nitro4-trifluoro-methylbenzyol)-1,3-cyclohexanedione (NTBC) in drinking water since birth to prevent the production of toxic metabolites and the onset of liver injury. Upon transplantation, NTBC is withdrawn to initiate liver injury ${ }^{140}$. In $\mathrm{Fah}^{-1-}$ mice, the regeneration of host hepatocytes, but not of donor cells, is impaired, which provides donor cells with a proliferative advantage ${ }^{140}$. Presumably, the regenerative signal-rich liver microenvironment facilitates the proliferation of donor cells.

In $\mathrm{Fah}^{-1-}$ mice transplanted with hepatocyte organoids, $\mathrm{FAH}^{+}$grafts of various sizes were observed, which most likely arose from clonal repopulation of engrafted cells (Fig. 4b) ${ }^{25}$. To track WNT activity in donor cells, hepatocytes derived from mice carrying the Axin2-rtTA;TetO$H 2 B: G F P$ reporter gene were transplanted into $\mathrm{Fah}^{-1-}$ mice. Two days prior to analysis, transplanted mice were administered doxycycline to activate Axin2 reporter expression in donor hepatocytes. GFP expression could be detected in the $\mathrm{FAH}^{+}$clones but not host cells. In smaller 


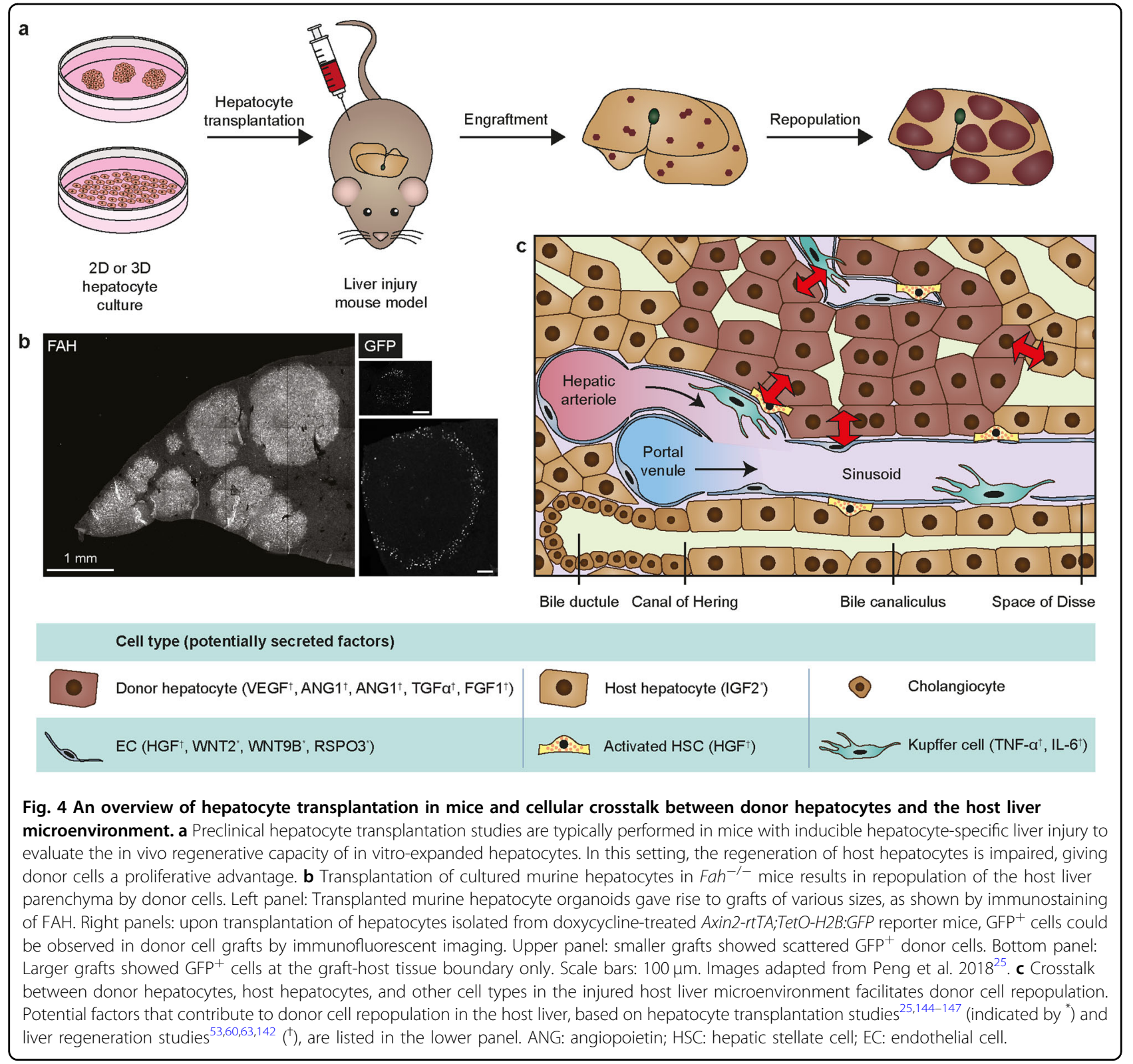

$\mathrm{FAH}^{+}$clones, many $\mathrm{GFP}^{+}$cells were observed scattered throughout the clones, indicating a response to WNT signaling (Fig. 4b). In contrast, in larger grafts, $\mathrm{GFP}^{+}$cells were restricted to donor cells at the graft-host tissue boundary and were absent from the center of the grafts (Fig. 4b). Consistent with this observation, the expression of Wnt2, Wnt9b, and Rspo3 was largely upregulated in host tissue ${ }^{25}$. Expression of RSPO3, which is normally restricted to endothelial cells around CVs in uninjured livers ${ }^{33,34}$, was upregulated in endothelial cells in the host tissue around the clonal boundary ${ }^{25}$. Likely, the WNTrich environment in the host liver facilitates donor cell proliferation, consistent with the role of WNT signaling in other regeneration models ${ }^{141}$.
Previous studies have shown that angiogenesis is a critical aspect of liver regeneration and function ${ }^{109,110,142}$. VEGF secreted by regenerating hepatocytes promotes LSEC proliferation. Neutralization of VEGF not only impaired the proliferation of endothelial cells but also indirectly impaired hepatocytes ${ }^{142}$. Further, it was demonstrated that LSECs establish an inductive vascular niche during liver regeneration after partial hepatectomy through activation of VEGF receptor 2 (VEGFR2) and upregulation of transcription factor inhibitor of DNA binding 1 (ID1), which results in the production of WNT2 and HGF to stimulate hepatocyte proliferation ${ }^{143}$. These observations are consistent with the crosstalk between hepatocytes and LSECs, which serve as a major source of 
WNT-RSPO signals. In addition to endothelial cellhepatocyte crosstalk, another example of paracrine signaling between host tissue and donor hepatocytes has been described. Upon transplantation of donor hepatocytes, IGF2 was upregulated by host hepatocytes to support liver repopulation. While blocking IGF2 in vivo impaired liver regeneration, its activation resulted in increased hepatocyte engraftment and numbers of MKI67-positive donor cells ${ }^{144,145}$. It is likely that a network of crosstalk between donor hepatocytes, host hepatocytes, and the host microenvironment dictates repopulation by donor hepatocytes (Fig. 4c).

The contribution of HSCs to hepatocyte engraftment has been studied in a preclinical transplantation setting using animal models that do not allow for selective repopulation of donor cells. Upon hepatocyte transplantation in DPPIV-deficient rats, increased numbers of host HSCs were observed posttransplantation, primarily in close proximity to engrafted $\mathrm{DPPIV}^{+}$donor cells, suggesting close interaction and potential crosstalk between HSCs and donor hepatocytes during donor cell engraftment $^{146}$. In a later study, primary human hepatocytes were cotransplanted with isolated human HSCs or an
HSC cell line (LX-2) into SCID mice ${ }^{147}$. Transplantation of human hepatocytes alone resulted in engraftment of scattered human $\mathrm{ALB}^{+}$cells throughout the parenchyma, whereas cotransplantation resulted in increased engraftment, which was more evident for LX-2 cells and culture-activated HSCs than for quiescent $\mathrm{HSCs}^{147}$. Together, these studies suggest that a close interaction between hepatocytes and HSCs is involved in the regulation of hepatocyte engraftment upon transplantation, although the exact mechanism and signaling pathways involved and the question of whether HSCs also contribute to supporting liver repopulation by donor cells remain to be elucidated. Although the role of HSCs during liver regeneration is not well understood, it is known that HSCs are activated in response to paracrine signals produced by regenerating hepatocytes and other cell types in the microenvironment (Kupffer cells and LSECs). In addition, HSCs are a main source of HGF, a driving mitogenic factor of hepatocyte proliferation for liver regeneration upon partial hepatectomy ${ }^{63}$. Possibly, similar crosstalk between HSCs and donor hepatocytes also contributes to donor cell engraftment and repopulation.

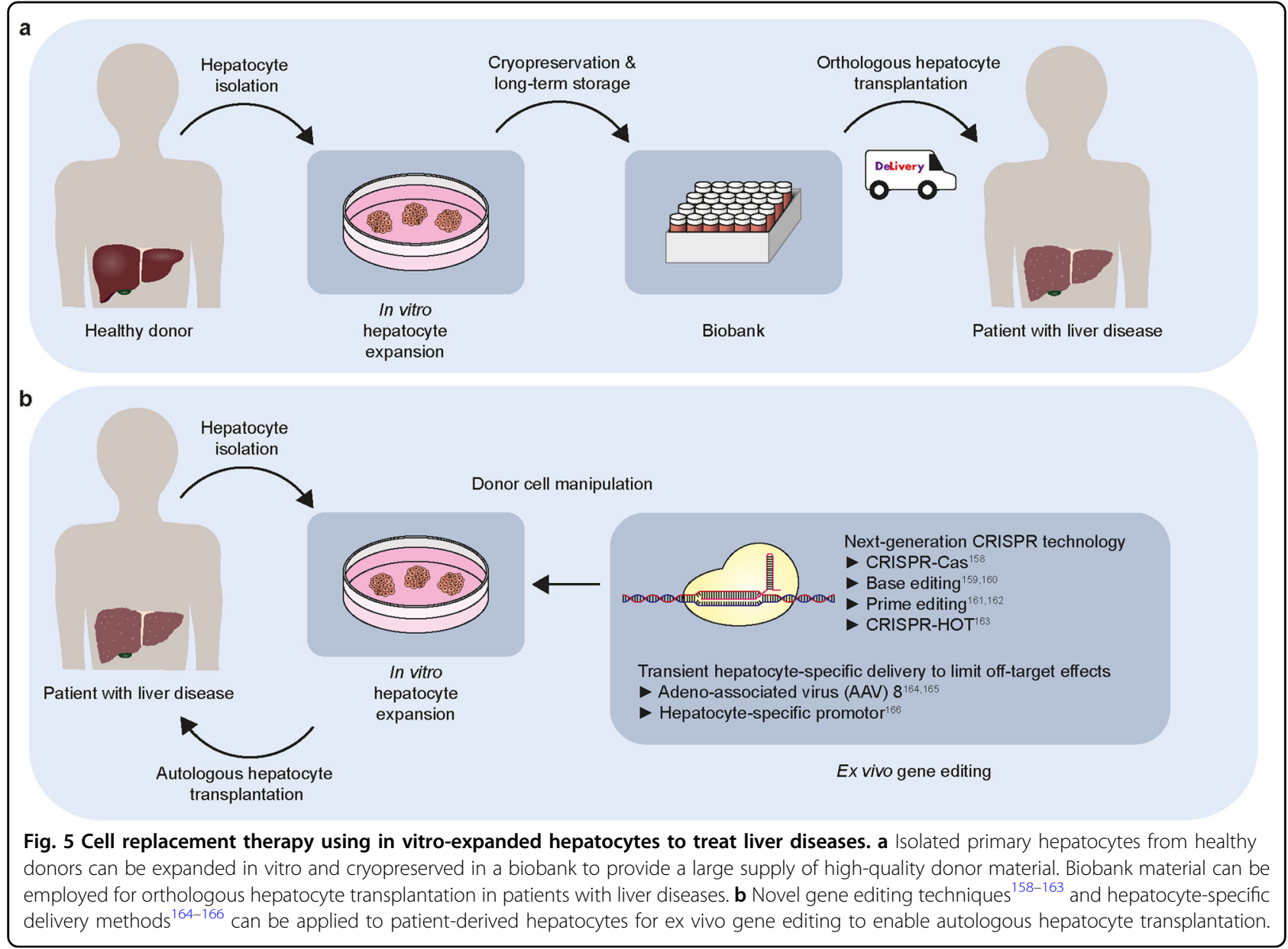


The role of Kupffer cells and other immune cells in hepatocyte transplantation is complicated, as they are largely involved in the clearance of transplanted cells and prevention of engraftment, which is beyond the scope of this review and has been discussed elsewhere ${ }^{148}$. On the other hand, Kupffer cells are known to be a prominent source of TNF- $\alpha$ and IL-6, two major inflammatory cytokines that are involved in liver regeneration ${ }^{63,78-80}$ and support the proliferation of hepatocytes in vitro ${ }^{25,81,86}$. Therefore, a supporting role for Kupffer cells during liver repopulation by transplanted hepatocytes in vivo could also be expected.

\section{Conclusions}

The ability to generate or expand the appropriate cell type in vitro, thus providing an abundant supply of healthy donor cells, is the first essential step in making cell replacement therapy feasible. Recent breakthroughs in expanding primary hepatocytes in vitro while retaining their in vivo regenerative capacity represent a major milestone towards enabling hepatocyte transplantation. Furthermore, the ability to expand, cryopreserve and transport hepatocytes without compromising their quality would enable the creation of a biobank that would ensure the availability of (in vitro-expanded) donor materials for hepatocyte transplantation, thus resolving the issue of donor shortage (Fig. 5a). More importantly, autologous cell transplantation will become feasible, as novel gene editing techniques and hepatocyte-specific delivery methods can be applied to patient-derived hepatocytes for ex vivo gene editing to restore disease-specific mutations (Fig. 5b). Although currently, the lack of long-term efficacy due to either insufficient engraftment or lack of long-term maintenance of donor cells in host livers may pose a major challenge in cell transplantation, we expect the understanding of the mechanisms through which donor hepatocytes repopulate the diseased host liver to provide insights into strategies for overcoming these limitations (Fig. 5b). We are optimistic that in vitro-expanded primary hepatocytes will be widely employed for cell replacement therapy to treat liver diseases in the foreseeable future.

\footnotetext{
Acknowledgements

We thank Kyle Loh (Stanford University) for the useful discussion and critical reading of the manuscript, as well as Iris Aullon Alcaine for her help with literature research and her support during writing.
}

\section{Conflict of interest}

The authors declare no competing interests.

\section{Publisher's note}

Springer Nature remains neutral with regard to jurisdictional claims in published maps and institutional affiliations.

Received: 9 November 2020 Revised: 11 January 2021 Accepted: 14 January 2021.

Published online: 18 October 2021

\section{References}

1. Hewitt, N. J. et al. Primary hepatocytes: current understanding of the regulation of metabolic enzymes and transporter proteins, and pharmaceutical practice for the use of hepatocytes in metabolism, enzyme induction, transporter, clearance, and hepatotoxicity studies. Drug Metab. Rev. 39, 159-234 (2008).

2. Acosta, D., Anuforo, D. C., McMillin, R., Soine, W. H. \& Smith, R. V. Comparison of cytochrome P-450 levels in adult rat liver, postnatal rat liver, and primary cultures of postnatal rat hepatocytes. Life Sci. 25, 1413-1418 (1979).

3. Guguen-Guillouzo, C. \& Guillouzo, A. General review on in vitro hepatocyte models and their applications. Methods Mol. Biol. 640, 1-40 (2010).

4. Bissell, D. M., Arenson, D. M., Maher, J. J. \& Roll, F. J. Support of cultured hepatocytes by a laminin-rich gel. Evidence for a functionally significant subendothelial matrix in normal rat liver. J. Clin. Invest. 79, 801-812 (1987).

5. Dunn, J. C. Y., Yarmush, M. L., Koebe, H. G. \& Tompkins, R. G. Hepatocyte function and extracellular matrix geometry: long-term culture in a sandwich configuration. Faseb J. 3, 174-177 (1989).

6. Guguen-Guillouzo, C. et al. Maintenance and reversibility of active albumin secretion by adult rat hepatocytes co-cultured with another liver epithelial cell type. Exp. Cell Res 143, 47-54 (1983).

7. Clement, B. et al. Long-term co-cultures of adult human hepatocytes with rat liver epithelial cells: modulation of albumin secretion and accumulation of extracellular material. Hepatology 4, 373-380 (1984).

8. Mesnil, M., Fraslin, J.-M., Piccoli, C., Yamasaki, H. \& Guguen-Guillouzo, C. Cell contact but not junctional communication (dye coupling) with biliary epithelial cells is required for hepatocytes to maintain differentiated functions. Exp. Cell Res. 173, 524-533 (1987).

9. Landry, J., Bernier, D., Ouellet, C., Goyette, R. \& Marceau, N. Spheroidal aggregate culture of rat liver cells: histotypic reorganization, biomatrix deposition, and maintenance of functional activities. J. Cell Biol. 101, 914-923 (1985).

10. Bachmann, A. et al. 3D cultivation techniques for primary human hepatocytes. Microarrays 4, 64-83 (2015).

11. Bell, C. C. et al. Comparison of hepatic 2D sandwich cultures and 3D spheroids for long-term toxicity applications: a multicenter study. Toxicol. Sci. 162, 655-666 (2018)

12. Lauschke, V. M., Shafagh, R. Z., Hendriks, D. F. G. \& Ingelman-Sundberg, M. 3D primary hepatocyte culture systems for analyses of liver diseases, drug metabolism, and toxicity: emerging culture paradigms and applications. Biotechnol. J. 14, 1800347 (2019).

13. Ang, L. T. et al. A roadmap for human liver differentiation from pluripotent stem cells. Cell Rep. 22, 2190-2205 (2018).

14. Si-Tayeb, K. et al. Highly efficient generation of human hepatocyte-like cells from induced pluripotent stem cells. Hepatology 51, 297-305 (2010).

15. Cai, J. et al. Directed differentiation of human embryonic stem cells into functional hepatic cells. Hepatology 45, 1229-1239 (2007).

16. Wang, Q. et al. Generation of human hepatocytes from extended pluripotent stem cells. Cell Res. 30, 810-813 (2020).

17. Maepa, S. W. \& Ndlovu, H. Advances in generating liver cells from pluripotent stem cells as a tool for modeling liver diseases. Stem Cells 38, 606-612 (2020).

18. Raju, R. et al. In vitro pluripotent stem cell differentiation to hepatocyte ceases further maturation at an equivalent stage of E15 in mouse embryonic liver development. Stem Cells Dev. 27, 910-921 (2018).

19. Sato, T. et al. Single Lgr5 stem cells build crypt-villus structures in vitro without a mesenchymal niche. Nature 459, 262-265 (2009).

20. Huch, M. et al. In vitro expansion of single Lgr5+ liver stem cells induced by Wnt-driven regeneration. Nature 494, 247-250 (2013).

21. Huch, M. et al. Long-term culture of genome-stable bipotent stem. Cells Adult Hum. Liver. Cell 160, 299-312 (2015).

22. Lugli, N. et al. R-spondin 1 and noggin facilitate expansion of resident stem cells from non-damaged gallbladders. EMBO Rep. 17, 769-779 (2016).

23. Sampaziotis, F. et al. Reconstruction of the mouse extrahepatic biliary tree using primary human extrahepatic cholangiocyte organoids. Nat. Med. 23, 954-963 (2017).

24. Rimland, C. A. et al. Regional differences in human biliary tissues and corresponding in vitro derived organoids. Hepatology https://doi.org/10.1002/ hep.31252 (2020).

25. Peng, W. C. et al. Inflammatory cytokine TNFa promotes the long-term expansion of primary hepatocytes in 3D culture. Cell 175, 1607-1619 (2018).

26. $\mathrm{Hu}, \mathrm{H}$. et al. Long-term expansion of functional mouse and human hepatocytes as 3D organoids. Cell 175, 1591-1606 (2018). 
27. MacDONALD, R. A. Lifespan of liver cells: autoradiographic study using tritiated thymidine in normal, cirrhotic, and partially hepatectomized rats. Arch Intern. Med. 107, 335-343 (1961).

28. Magami, Y. et al. Cell proliferation and renewal of normal hepatocytes and bile duct cells in adult mouse liver. Liver 22, 419-425 (2002).

29. Arrojo e Drigo, R. et al. Age mosaicism across multiple scales in adult tissues. Cell Metab. 30, 343-351 (2019).

30. Kudryavtsev, B. N., Kudryavtseva, M. V., Sakuta, G. A. \& Stein, G. I. Human hepatocyte polyploidization kinetics in the course of life cycle. Virchows Arch. B 64, 387 (1993).

31. Miyajima, A., Tanaka, M. \& Itoh, T. Stem/progenitor cells in liver development, homeostasis, regeneration, and reprogramming. Cell Stem Cell 14, 561-574 (2014).

32. Wang, B., Zhao, L., Fish, M., Logan, C. Y. \& Nusse, R. Self-renewing diploid Axin2+ cells fuel homeostatic renewal of the liver. Nature 524, 180-185 (2015)

33. Rocha, A. S. et al. The angiocrine factor Rspondin3 is a key determinant of liver zonation. Cell Rep. 13, 1757-1764 (2015).

34. Planas-Paz, L. et al. The RSPO-LGR4/5-ZNRF3/RNF43 module controls liver zonation and size. Nat. Cell Biol. 18, 467-479 (2016).

35. Zhao, L. et al. Tissue repair in the mouse liver following acute carbon tetrachloride depends on injury-induced Wnt/ß-catenin signaling. Hepatology 69, 2623-2635 (2019).

36. Tan, X., Behari, J., Cieply, B., Michalopoulos, G. K. \& Monga, S. P. S. Conditional deletion of $\beta$-catenin reveals its role in liver growth and regeneration. Gastroenterology 131, 1561-1572 (2006).

37. Monga, S. P. $\beta$-catenin signaling and roles in liver homeostasis, injury, and tumorigenesis. Gastroenterology 148, 1294-1310 (2015).

38. Cairo, S. et al. Hepatic stem-like phenotype and interplay of Wnt/ß-catenin and myc signaling in aggressive childhood liver cancer. Cancer Cell 14, 471-484 (2008).

39. Gröbner, S. N. et al. The landscape of genomic alterations across childhood cancers. Nature 555, 321-327 (2018)

40. Clevers, H., Loh, K. M. \& Nusse, R. An integral program for tissue renewal and regeneration: Wnt signaling and stem cell control. Science 346, 1248012 (2014).

41. Lau, W., de, Peng, W. C., Gros, P. \& Clevers, H. The R-spondin/Lgr5/Rnf43 module: regulator of Wnt signal strength. Gene Dev. 28, 305-316 (2014).

42. Lin, S. et al. Distributed hepatocytes expressing telomerase repopulate the liver in homeostasis and injury. Nature 556, 244-248 (2018).

43. Nault, J. C. et al. High frequency of telomerase reverse-transcriptase promoter somatic mutations in hepatocellular carcinoma and preneoplastic lesions. Nat. Commun. 4, 2218 (2013).

44. Ang, C. H. et al. Lgr5+ pericentral hepatocytes are self-maintained in normal liver regeneration and susceptible to hepatocarcinogenesis. Proc. Natl Acad. Sci. USA 116, 19530-19540 (2019).

45. Chen, F. et al. Broad distribution of hepatocyte proliferation in liver homeostasis and regeneration. Cell Stem Cell 26, 27-33 (2019).

46. Matsumoto, T., Wakefield, L., Tarlow, B. D. \& Grompe, M. In vivo lineage tracing of polyploid hepatocytes reveals extensive proliferation during live regeneration. Cell Stem Cell 26, 34-47 (2019).

47. Sun, $T$. et al. AXIN2+pericentral hepatocytes have limited contributions to liver homeostasis and regeneration. Cell Stem Cell 26, 97-107 (2019).

48. Font-Burgada, J. et al. Hybrid periportal hepatocytes regenerate the injured liver without giving rise to cancer. Cell 162, 766-779 (2015).

49. Pek, N. M. Q., Liu, K. J., Nichane, M. \& Ang, L. T. Controversies surrounding the origin of hepatocytes in adult livers and the in vitro generation or propagation of hepatocytes. Cell Mol. Gastroenterology Hepatology https:/doi.org/ 10.1016/j.jcmgh.2020.09.016 (2020).

50. Heinke, P. et al. Diploid hepatocytes drive physiological liver renewal in adult humans. Biorxiv https://doi.org/10.1101/2020.08.07.230086 (2020).

51. Taub, R. Liver regeneration: from myth to mechanism. Nat. Rev. Mol. Cell Biol. 5, 836-847 (2004).

52. Michalopoulos, G. K. Hepatostat: liver regeneration and normal liver tissue maintenance. Hepatology 65, 1384-1392 (2017).

53. Michalopoulos, G. K. Liver regeneration. J. Cell Physiol. 213, 286-300 (2007)

54. Bucher, M. L. \& Swaffield, M. N. Regulation of hepatic regeneration in rats by synergistic action of insulin and glucagon. Proc. Natl Acad. Sci. USA72, 1157-1160 (1975).

55. Michalopoulos, G. K. Liver regeneration after partial hepatectomy critical analysis of mechanistic dilemmas. Am. J. Pathol. 176, 2-13 (2010).
56. Marubashi, S. et al. Effect of portal hemodynamics on liver regeneration studied in a novel portohepatic shunt rat model. Surgery 136, 1028-1037 (2004)

57. Niehrs, C. \& Acebron, S. P. Mitotic and mitogenic Wnt signalling. Embo J. 31, 2705-2713 (2012).

58. Peng, W. C. et al. Structure of stem cell growth factor R-spondin 1 in complex with the ectodomain of its receptor LGR5. Cell Rep. 3, 1885-1892 (2013).

59. Peng, W. C. et al. Structures of Wnt-Antagonist ZNRF3 and its complex with R-Spondin 1 and implications for signaling. PLOS ONE 8, e83110 (2013).

60. Ding, B.-S. et al. Inductive angiocrine signals from sinusoidal endothelium are required for liver regeneration. Nature 468, 310-315 (2010).

61. Cerami, A. Inflammatory cytokines. Clin. Immunol. Immunop 62, S3-S10 (1992).

62. Cornell, R. P. Gut-derived endotoxin elicits hepatotrophic factor secretion for liver regeneration. Am. J. Physiol.-regulatory Integr. Comp. Physiol. 249, R551-R562 (1985).

63. Michalopoulos, G. K. \& Bhushan, B. Liver regeneration: biological and pathological mechanisms and implications. Nat. Rev. Gastroenterol. https:// doi.org/10.1038/s41575-020-0342-4 (2020).

64. Tsai, S.-M. \& Wang, W.-P. Expression and function of fibroblast growth factor (FGF) 7 during liver regeneration. Cell Physiol. Biochem. 27, 641-652 (2011).

65. Kong, B. et al. Fibroblast growth factor 15 deficiency impairs liver regeneration in mice. Am. J. Physiol.-gastr L 306, G893-G902 (2014).

66. Sugimoto, $H$. et al. BMP-7 functions as a novel hormone to facilitate liver regeneration. FASEB J. 21, 256-264 (2007).

67. Michalopoulos, G. K. In The Liver: Biology and Pathobiology 6th edn (eds Arias, I. M. et al.) Ch. 45 (Wiley-Blackwell, Hoboken, 2020).

68. Yimlamai, D., Fowl, B. H. \& Camargo, F. D. Emerging evidence on the role of the Hippo/YAP pathway in liver physiology and cancer. J. Hepatol. 63, 1491-1501 (2015).

69. Patel, S. H., Camargo, F. D. \& Yimlamai, D. Hippo signaling in the liver regulates organ size, cell fate, and carcinogenesis. Gastroenterology $\mathbf{1 5 2}$ 533-545 (2017).

70. Walker, P. R. \& Potter, V. R. Isozyme studies on adult, regenerating, precancerous and developing liver in relation to findings in hepatomas. Adv. Enzym. Regul. 10, 339-364 (1972).

71. Bonney, R. J., Hopkins, H. A., Walker, P. R. \& Potter, V. R. Glycolytic isoenzymes and glycogen metabolism in regenerating liver from rats on controlled feeding schedules. Biochem J. 136, 115-124 (1973).

72. Campbell, J. S. et al. Expression of suppressors of cytokine signaling during liver regeneration. J. Clin. Invest. 107, 1285-1292 (2001).

73. Brand, S. et al. IL-22-mediated liver cell regeneration is abrogated by SOCS-1/ 3 overexpression in vitro. Am. J. Physiol.-Gastr. L 292, G1019-G1028 (2007).

74. Sun, R. \& Gao, B. Negative regulation of liver regeneration by innate immunity (natural killer cells/interferon-ү). Gastroenterology 127, 1525-1539 (2004).

75. Katsuda, T. et al. Conversion of terminally committed hepatocytes to culturable bipotent progenitor cells with regenerative capacity. Cell Stem Cell 20, 41-55 (2017).

76. Broutier, L. et al. Human primary liver cancer-derived organoid cultures for disease modeling and drug screening. Nat. Med. 23, 1424-1435 (2017).

77. Mitaka, T., Mikami, M., Sattler, G. L., Pitot, H. C. \& Mochizuki, Y. Small cell colonies appear in the primary culture of adult rat hepatocytes in the presence of nicotinamide and epidermal growth factor. Hepatology 16, 440-447 (1992).

78. Akerman, P. et al. Antibodies to tumor necrosis factor-alpha inhibit liver regeneration after partial hepatectomy. Am. J. Physiol.-Gastr L 263, G579-G585 (1992)

79. Cressman, D. E. et al. Liver failure and defective hepatocyte regeneration in interleukin-6-deficient mice. Science 274, 1379-1383 (1996).

80. Yamada, Y., Kirillova, I., Peschon, J. J. \& Fausto, N. Initiation of liver growth by tumor necrosis factor: deficient liver regeneration in mice lacking type I tumor necrosis factor receptor. Proc. Natl Acad. Sci. USA 94, 1441-1446 (1997).

81. Sérandour, A. et al. TNFa-mediated extracellular matrix remodeling is required for multiple division cycles in rat hepatocytes. Hepatology $\mathbf{4 1}$ 478-486 (2005).

82. Kamiya, A. \& Gonzalez, F. J. TNF-a regulates mouse fetal hepatic maturation induced by oncostatin M and extracellular matrices. Hepatology 40, 527-536 (2004).

83. Beg, A. A. \& Baltimore, D. An essential role for NF-kB in preventing TNF-ainduced cell death. Science 274, 782-784 (1996). 
84. Papa, S., Bubici, C., Zazzeroni, F. \& Franzoso, G. Mechanisms of liver disease: cross-talk between the NF-KB and JNK pathways. Biol. Chem. 390, 965-976 (2009).

85. Ma, B. \& Hottiger, M. O. Crosstalk between Wnt/ß-Catenin and NF-kB Signaling Pathway during Inflammation. Front Immunol. 7, 378 (2016).

86. Hyun, J. et al. Epithelial splicing regulatory protein 2-mediated alternative splicing reprograms hepatocytes in severe alcoholic hepatitis. J. Clin. Invest 130, 2129-2145 (2020)

87. Aurora, A. B. \& Olson, E. N. Immune modulation of stem cells and regeneration. Cell Stem Cell 15, 14-25 (2014)

88. Karin, M. \& Clevers, H. Reparative inflammation takes charge of tissue regeneration. Nature 529, 307-315 (2016).

89. $\mathrm{Fu}, \mathrm{X}$. et al. Combination of inflammation-related cytokines promotes longterm muscle stem cell expansion. Cell Res 25, 655-673 (2015).

90. Katsura, H., Kobayashi, Y., Tata, P. R. \& Hogan, B. L. M. IL-1 and TNFa contribute to the inflammatory niche to enhance alveolar regeneration. Stem Cell Rep. 12, 657-666 (2019)

91. Choi, J. et al. Inflammatory signals induce AT2 cell-derived damage-associated transient progenitors that mediate alveolar regeneration. Cell Stem Cell 27, 366-382 (2020)

92. Takase, H. M. et al. FGF7 is a functional niche signal required for stimulation of adult liver progenitor cells that support liver regeneration. Gene Dev. 27 169-181 (2013).

93. Lázaro, C. A. et al. Establishment, characterization, and long-term maintenance of cultures of human fetal hepatocytes. Hepatology 38, 1095-1106 (2003)

94. $\mathrm{Ng}, \mathrm{S}$. S. et al. Long-term culture of human liver tissue with advanced hepatic functions. Jci Insight 2, e90853 (2017).

95. Kim, Y. et al. Small molecule-mediated reprogramming of human hepatocytes into bipotent progenitor cells. J. Hepatol. 70, 97-107 (2018).

96. Zhang, $\mathrm{K}$. et al. In vitro expansion of primary human hepatocytes with efficient liver repopulation capacity. Cell Stem Cell 23, 806-819 (2018).

97. Xiang, $C$. et al. Long-term functional maintenance of primary human hepatocytes in vitro. Science 364, 399-402 (2019)

98. Fu, G.-B. et al. Expansion and differentiation of human hepatocyte-derived liver progenitor-like cells and their use for the study of hepatotropic pathogens. Cell Res 29, 8-22 (2019).

99. Unzu, C. et al. Pharmacological induction of a progenitor state for the efficient expansion of primary human hepatocytes. Hepatology 69, 2214-2231 (2019).

100. Katsuda, T. et al. Generation of human hepatic progenitor cells with regenerative and metabolic capacities from primary hepatocytes. Elife $\mathbf{8}$ e47313 (2019).

101. Walldorf, J. et al. Expanding hepatocytes in vitro before cell transplantation: donor age-dependent proliferative capacity of cultured human hepatocytes. Scand. J. Gastroentero 39, 584-593 (2009).

102. Michalopoulos, G. K., Bowen, W. C., Mulè, K. Lopez-Talavera, J. C. \& Mars, W. Hepatocytes undergo phenotypic transformation to biliary epithelium in organoid cultures. Hepatology 36, 278-283 (2002).

103. Michalopoulos, G. K., Barua, L. \& Bowen, W. C. Transdifferentiation of rat hepatocytes into biliary cells after bile duct ligation and toxic biliary injury. Hepatology 41, 535-544 (2005).

104. Yanger, K. et al. Robust cellular reprogramming occurs spontaneously during liver regeneration. Gene Dev. 27, 719-724 (2013).

105. Schaub, J. R. et al. De novo formation of the biliary system by TGF $\beta$-mediated hepatocyte transdifferentiation. Nature 557, 247-251 (2018).

106. Costa, R. H., Kalinichenko, V. V., Holterman, A. L. \& Wang, X. Transcription factors in liver development, differentiation, and regeneration. Hepatology $\mathbf{3 8}$ 1331-1347 (2003).

107. Su, T. et al. Two-signal requirement for growth-promoting function of Yap in hepatocytes. Elife 4, e02948 (2015).

108. Michalopoulos, G. K. \& DeFrances, M. C. Liver regeneration. Science $\mathbf{2 7 6}$ 60-66 (1997).

109. Shimizu, H. et al. Vascular endothelial growth factor secreted by replicating hepatocytes induces sinusoidal endothelial cell proliferation during regeneration after partial hepatectomy in rats. J. Hepatol. 34, 683-689 (2001).

110. Shimizu, H. et al. Vascular endothelial growth factor and angiopoietins regulate sinusoidal regeneration and remodeling after partial hepatectomy in rats. World J. Gastroenterol. 11, 7254-7260 (2005).

111. Chembazhi, U. V., Bangru, S., Hernaez, M. \& Kalsotra, A. Cellular plasticity balances the metabolic and proliferation dynamics of a regenerating liver Biorxiv https://doi.org/10.1101/2020.05.29.124263 (2020).
112. Schaum, N. et al. Single-cell transcriptomics of 20 mouse organs creates a Tabula Muris. Nature 562, 367-372 (2018)

113. Halpern, K. B. et al. Single-cell spatial reconstruction reveals global division of labour in the mammalian liver. Nature 542, 352-356 (2017)

114. Benhamouche, S. et al. Apc tumor suppressor gene is the "Zonation-Keeper" of mouse liver. Dev. Cell 10, 759-770 (2006).

115. Hailfinger, S., Jaworski, M., Braeuning, A., Buchmann, A. \& Schwarz, M. Zonal gene expression in murine liver: lessons from tumors. Hepatology $\mathbf{4 3}$, 407-414 (2006).

116. Cheng, X. et al. Glucagon contributes to liver zonation. Proc. Natl Acad. Sci. USA 115, E4111-E4119 (2018).

117. Michalopoulos, G. K., Bowen, W. C., Mulé, K. \& Luo, J. HGF-, EGF-, and dexamethasone-induced gene expression patterns during formation of tissue in hepatic organoid cultures. Gene Expr. 11, 55-75 (2003).

118. Jungermann, K. \& Keitzmann, T. Zonation of parenchymal and nonparenchymal metabolism in liver. Annu Rev. Nutr. 16, 179-203 (1996).

119. Halpern, K. B. et al. Paired-cell sequencing enables spatial gene expression mapping of liver endothelial cells. Nat. Biotechnol. 36, 962-970 (2018).

120. Janda, C. Y. et al. Surrogate Wnt agonists that phenocopy canonical Wnt and $\beta$-catenin signalling. Nature 545, 234-237 (2017)

121. Miao, Y. et al. Next-generation surrogate Wnts support organoid growth and deconvolute frizzled pleiotropy in vivo. Cell Stem Cell 27, 840-851 (2020).

122. Habib, S. J. et al. A localized Wnt signal orients asymmetric stem cell division in vitro. Science 339, 1445-1448 (2013).

123. Lowndes, M., Rotherham, M., Price, J. C., El Haj, A. J. \& Habib, S. J. Immobilized WNT proteins act as a stem cell niche for tissue engineering. Stem Cell Rep. 7 126-137 (2016).

124. Lowndes, M., Junyent, S. \& Habib, S. J. Constructing cellular niche properties by localized presentation of Wht proteins on synthetic surfaces. Nat. Protoc. 12, 1498-1512 (2017).

125. Brassard, J. A., Nikolaev, M., Hübscher, T., Hofer, M. \& Lutolf, M. P. Recapitulating macro-scale tissue self-organization through organoid bioprinting. Nat Mater. https://doi.org/10.1038/s41563-020-00803-5 (2020).

126. Schneider, A. et al. Hepatocyte transplantation in an acute liver failure due to mushroom poisoning. Transplantation 82, 1115-1116 (2006).

127. Dhawan, A. et al. Hepatocyte transplantation for inherited factor VII deficiency. Transplantation 78, 1812-1814 (2004).

128. Fox, I. J. et al. Treatment of the Crigler-Najjar syndrome type I with hepatocyte transplantation. N. Engl. J. Med. 338, 1422-1427 (1998).

129. Ambrosino, G. et al. Isolated hepatocyte transplantation for Crigler-Najjar syndrome type 1. Cell Transpl. 14, 151-157 (2005).

130. Lysy, P. A. et al. Liver cell transplantation for Crigler-Najjar syndrome type I: Update and perspectives. World J. Gastroenterol 14, 3464-3470 (2008).

131. Barahman, M. et al. Hepatocyte transplantation: Quo Vadis? Int J. Radiat Oncol. Biol. Phys. 103, 922-934 (2018).

132. Soltys, K. A. et al. Host conditioning and rejection monitoring in hepatocyte transplantation in humans. J. Hepatol. 66, 987-1000 (2017).

133. Terry, C., Dhawan, A., Mitry, R. R. \& Hughes, R. D. Cryopreservation of isolated human hepatocytes for transplantation: state of the art. Cryobiology $\mathbf{5 3}$ 149-159 (2006).

134. Gómez-Lechón, M. J., Lahoz, A., Jiménez, N., Castell, J. V. \& Donato, M. T. Cryopreservation of rat, dog and human hepatocytes: influence of preculture and cryoprotectants on recovery, cytochrome P450 activities and induction upon thawing. Xenobiotica 36, 457-472 (2008)

135. Tarlow, B. D., Finegold, M. J. \& Grompe, M. Clonal tracing of Sox9+ liver progenitors in mouse oval cell injury. Hepatology 60, 278-289 (2014).

136. Yanger, $\mathrm{K}$. et al. Adult hepatocytes are generated by self-duplication rather than stem cell differentiation. Cell Stem Cell 15, 340-349 (2014).

137. Raven, A. et al. Cholangiocytes act as facultative liver stem cells during impaired hepatocyte regeneration. Nature 547, 350-354 (2017).

138. Grompe, M. et al. Loss of fumarylacetoacetate hydrolase is responsible for the neonatal hepatic dysfunction phenotype of lethal albino mice. Genes Dev. 7 2298-2307 (1993).

139. Azuma, H. et al. Robust expansion of human hepatocytes in $\mathrm{Fah}^{-1-} / \mathrm{Rag}^{-1-}$ $/ / 12 \mathrm{rg}^{-1-}$ mice. Nat. Biotechnol. 25, 903-910 (2007).

140. Overturf, K. et al. Hepatocytes corrected by gene therapy are selected in vivo in a murine model of hereditary tyrosinaemia type I. Nat. Genet 12, 266-273 (1996).

141. Russell, J. O. Wnt/B-catenin signaling in liver development, homeostasis, and pathobiology. Annu. Rev. Pathol. 13, 351-378 (2018). 
142. Taniguchi, E., Sakisaka, S., Matsuo, K., Tanikawa, K. \& Sata, M. Expression and Role of Vascular Endothelial Growth Factor in Liver Regeneration After Partial Hepatectomy in Rats. J. Histochem Cytochem 49, 121-129 (2000).

143. Ding, J. et al. Spontaneous hepatic repopulation in transgenic mice expressing mutant human a1-antitrypsin by wild-type donor hepatocytes. J. Clin. Invest 121, 1930-1934 (2011)

144. Wang, M.-J. et al. Insulin-like growth factor 2 is a key mitogen driving liver repopulation in mice. Cell Death Dis. 9, 26 (2018).

145. Liu, J. et al. Pericentral hepatocytes produce insulin-like growth factor-2 to promote liver regeneration during selected injuries in mice. Hepatology 66 2002-2015 (2017).

146. Benten, D. et al. Hepatocyte transplantation activates hepatic stellate cells with beneficial modulation of cell engraftment in the rat. Hepatology $\mathbf{4 2}$ 1072-1081 (2005).

147. Dusabineza, A.-C. et al. Hepatic stellate cells improve engraftment of human primary hepatocytes: a preclinical transplantation study in an animal model. Cell Transpl. 24, 2557-2571 (2014).

148. Oldhafer, F., Bock, M., Falk, C. S. \& Vondran, F. W. R. Immunological aspects of liver cell transplantation. World J. Transpl. 6, 42 (2016).

149. Nagy, P., Thorgeirsson, S. S. \& Grisham, J. W. In The Liver: Biology and Pathobiology 6th edn (eds Arias, I. M. et al.) Ch. 1 (Wiley-Blackwell, Hoboken, 2020).

150. Guha, C. et al. Normal hepatocytes correct serum bilirubin after repopulation of Gunn rat liver subjected to irradiation/partial resection. Hepatology 36, 354-362 (2002).

151. Yamanouchi, K. et al. Hepatic irradiation augments engraftment of donor cells following hepatocyte transplantation. Hepatology 49, 258-267 (2009).

152. Grompe, M. \& Al-Dhalimy, M. Mutations of the fumarylacetoacetate hydrolase gene in four patients with tyrosinemia, Type I. Hum. Mutat. 2, 85-93 (1993).

153. Lomas, D. A., LI-Evans, D., Finch, J. T. \& Carrell, R. W. The mechanism of Z a1antitrypsin accumulation in the liver. Nature 357, 605-607 (1992).
154. Bilir, B. M. et al. Hepatocyte transplantation in acute liver failure. Liver Transpl. 6, 32-40 (2000).

155. Bernal, W., Auzinger, G., Dhawan, A. \& Wendon, J. Acute liver failure. Lancet 376, 190-201 (2010).

156. Rela, M., Kaliamoorthy, I. \& Reddy, M. S. Current status of auxiliary partial orthotopic liver transplantation for acute liver failure. Liver Transpl. 22, 1265-1274 (2016).

157. Fisher, R. A. \& Strom, S. C. in The Hepatocyte Review (M. N. Berry, A. M. Edwards eds) Ch. 28 (Kluwer Academic Publishers, Amsterdam, 2000)

158. Guan, Y. et al. Human hepatic organoids for the analysis of human genetic diseases. JCl Insight 2, e94954 (2017).

159. Komor, A. C., Kim, Y. B., Packer, M. S., Zuris, J. A. \& Liu, D. R. Programmable editing of a target base in genomic DNA without double-stranded DNA cleavage. Nature 533, 420-424 (2016).

160. Rees, H. A. \& Liu, D. R. Base editing: precision chemistry on the genome and transcriptome of living cells. Nat. Rev. Genet. 19, 770-788 (2018).

161. Anzalone, A. V. et al. Search-and-replace genome editing without doublestrand breaks or donor DNA. Nature 576, 149-157 (2019).

162. Marzec, M., Brąszewska-Zalewska, A. \& Hensel, G. rime editing: a new way for genome editing. Trends Cell Biol. 30, 257-259 (2020).

163. Artegiani, B. et al. Fast and efficient generation of knock-in human organoids using homology-independent CRISPR-Cas9 precision genome editing. Nat. Cell Biol. 22, 321-331 (2020).

164. VanLith, C. J. et al. Ex vivo hepatocyte reprograming promotes homologydirected DNA repair to correct metabolic disease in mice after transplantation. Hepatol. Commun. 3, 558-573 (2019).

165. Levy, J. M. et al. Cytosine and adenine base editing of the brain, liver, retina, heart and skeletal muscle of mice via adeno-associated viruses. Nat. Biomed. Eng. 4, 97-110 (2020).

166. Murillo, O. et al. Gene therapy of Wilson disease: A "golden" opportunity using rAAV on the 50th anniversary of the discovery of the virus. J. Hepatol. 64, 265-267 (2016). 